\title{
Transcriptional and Morpho-Physiological Responses of Marchantia polymorpha upon Phosphate Starvation
}

\author{
Félix Rico-Reséndiz ${ }^{1}{ }^{\circledR}$, Sergio Alan Cervantes-Pérez ${ }^{2}$, Annie Espinal-Centeno ${ }^{1}$, \\ Melissa Dipp-Álvarez ${ }^{1}{ }^{\circledR}$, Araceli Oropeza-Aburto ${ }^{2}$, Enrique Hurtado-Bautista ${ }^{3}$, \\ Andrés Cruz-Hernández ${ }^{4}$, John L. Bowman ${ }^{5}$, Kimitsune Ishizaki ${ }^{6}{ }^{\mathbb{D}}$, \\ Mario A. Arteaga-Vázquez ${ }^{7}\left(\mathbb{D}\right.$, Luis Herrera-Estrella ${ }^{2,8}$ (D) and Alfredo Cruz-Ramírez ${ }^{1, *}$ \\ 1 Molecular and Developmental Complexity Group, Unidad de Genómica Avanzada, \\ Centro de Investigación y Estudios Avanzados, Instituto Politécnico Nacional, \\ Irapuato 36824, Guanajuato, Mexico; edgardo.rico@cinvestav.mx (F.R.-R.); \\ annie.espinal@cinvestav.mx (A.E.-C.); melissa.dipp@cinvestav.mx (M.D.-Á.) \\ 2 Plant Physiology and Metabolic Engineering Group, Unidad de Genómica Avanzada, \\ Centro de Investigación y Estudios Avanzados, Instituto Politécnico Nacional, \\ Irapuato 36824, Guanajuato, Mexico; sergio.cervantes@cinvestav.mx (S.A.C.-P.); \\ araceli.oropeza@cinvestav.mx (A.O.-A.); lherrerae@cinvestav.mx (L.H.-E.) \\ 3 Molecular Biology and Microbial Ecology, Unidad Irapuato, Centro de Investigación y Estudios Avanzados, \\ Instituto Politécnico Nacional, Irapuato 36824, Guanajuato, Mexico; enrique.hurtado@cinvestav.mx \\ 4 Escuela de Agronomía, Universidad de La Salle Bajío, León 37160, Guanajuato, Mexico; \\ acruz@delasalle.edu.mx \\ 5 School of Biological Sciences, Monash University, Melbourne, Victoria 3800, Australia; \\ john.bowman@monash.edu \\ 6 Graduate School of Science, Kobe University, Kobe 657-8501, Japan; kimi@emerald.kobe-u.ac.jp \\ 7 Group of Epigenetics and Developmental Biology, Instituto de Biotecnología y Ecología Aplicada (INBIOTECA), \\ Universidad Veracruzana, Xalapa 91640, Mexico; maarteaga@uv.mx \\ 8 Institute of Genomics for Crop Abiotic Stress Tolerance, Department of Plant and Soil Sciences, \\ Texas Tech University, Lubbock, TX 79409, USA \\ * Correspondence: alfredo.cruz@cinvestav.mx; Tel.: +52-462-166-3000 (ext. 3005)
}

Received: 10 October 2020; Accepted: 30 October 2020; Published: 7 November 2020

\begin{abstract}
Phosphate (Pi) is a pivotal nutrient that constraints plant development and productivity in natural ecosystems. Land colonization by plants, more than 470 million years ago, evolved adaptive mechanisms to conquer Pi-scarce environments. However, little is known about the molecular basis underlying such adaptations at early branches of plant phylogeny. To shed light on how early divergent plants respond to Pi limitation, we analyzed the morpho-physiological and transcriptional dynamics of Marchantia polymorpha upon Pi starvation. Our phylogenomic analysis highlights some gene networks present since the Chlorophytes and others established in the Streptophytes (e.g., PHR1-SPX1 and STOP1-ALMT1, respectively). At the morpho-physiological level, the response is characterized by the induction of phosphatase activity, media acidification, accumulation of auronidins, reduction of internal Pi concentration, and developmental modifications of rhizoids. The transcriptional response involves the induction of MpPHR1, Pi transporters, lipid turnover enzymes, and MpMYB14, which is an essential transcription factor for auronidins biosynthesis. MpSTOP2 up-regulation correlates with expression changes in genes related to organic acid biosynthesis and transport, suggesting a preference for citrate exudation. An analysis of MpPHR1 binding sequences (P1BS) shows an enrichment of this cis regulatory element in differentially expressed genes. Our study unravels the strategies, at diverse levels of organization, exerted by M. polymorpha to cope with low Pi availability.
\end{abstract}

Keywords: land plant evolution; Marchantia polymorpha; Pi starvation and RNA-seq 


\section{Introduction}

The colonization of land by plants was a complex ecological, evolutionary, and developmental (eco-evo-devo) process that occurred around 470 million years ago [1,2]. Several developmental innovations, which allowed early plants to adapt and survive on terrestrial environments, have been deeply reviewed elsewhere [3-5]. However, little is known about the molecular innovations that allowed plants to thrive in conditions of low nutrient availability. The molecular mechanisms underlying the morphological and physiological strategies to forage low available nutrients have been mostly described in diverse plant root systems. This is the case for most studies focused on describing the adaptation of plants to phosphate starvation [6-8]. Phosphorus is an essential macronutrient for proper plant development, which can only be acquired as ortho-phosphate (Pi) by plant roots [9]. In natural ecosystems, $\mathrm{Pi}$ is generally distributed at low concentrations due to the rapid formation of inorganic complexes with $\mathrm{Ca}^{2+}$ or $\mathrm{Al}^{3+}$ and $\mathrm{Fe}^{3+}$, under alkaline or acid conditions, respectively [10,11]. This phenomenon causes up to $70 \%$ of global agricultural areas to display a limited Pi availability, affecting both plant development and crop productivity [12]. To enhance Pi uptake and recycling, diverse molecular mechanisms underlying plant morphological and physiological strategies are induced [7,8]. In Arabidopsis thaliana, these strategies have been characterized and classified in local and systemic responses [13]. The local response largely depends on the contact of the primary root tip with a Pi-depleted substratum, whose final outcome is the full differentiation of the primary root meristem and the triggering of lateral root development [14]. One of the early events that takes place in the local response of Arabidopsis to this stress involves the accumulation of the protein SENSITIVE TO PROTON RHIZOTOXICITY 1 (AtSTOP1), a zinc finger transcription factor (TF) [15]. AtSTOP1 promotes the expression of several genes including the ALUMINIUM ACTIVATED MALATE TRANSPORTER 1 (AtALMT1), which encodes a malic acid efflux transporter $[15,16]$. Malate efflux contributes to the modification of the iron distribution and impacts directly the Arabidopsis root system architecture (RSA) under low Pi availability, $\mathrm{Al}^{3+}$ toxicity, and acid $\mathrm{pH}[15,16]$. AtSTOP1 induces the expression of REGULATION OF ALMT1 EXPRESSION 1 (AtRAE1), which encodes an F-Box protein that ubiquitinates STOP1, which is degraded via the 26S proteasome pathway [17]. Additionally, exuded organic acids (OAs) release Pi from complexes with $\mathrm{Al}^{3+}$ or $\mathrm{Fe}^{3+}$ [18]. However, the post-translational regulation of AtSTOP1, in both high and low Pi conditions, remains elusive. As part of the local response, the multicopper oxidase LOW PHOSPHATE ROOT 1 (AtLPR1) changes the iron oxidation state from $\mathrm{Fe}^{2+}$ to $\mathrm{Fe}^{3+}$ in the root tip apoplast under low Pi conditions, this phenomenon correlates with callose accumulation and the process of differentiation of the root apical meristem (RAM) [19]. Iron redistribution and redox fluctuations, coupled to reactive oxygen species (ROS) signaling, promote the expression of the CLAVATA3/ENDOSPERM SURROUNDING REGION 14 (AtCLE14) gene. AtCLE14 triggers the full differentiation of the RAM via a signaling pathway that PEPTIDE RECEPTOR 2 (AtPEPR2) and CLAVATA 2 (AtCLV2) receptors use, which, in turn, may act negatively upstream of POLTERGEIST (AtPOL) and POLTERGEIST-LIKE 1 (AtPLL1), SCARECROW (AtSCR), and SHORT ROOT (AtSHR) [20,21]. The systemic response to low Pi availability is controlled by the internal concentration of inositol polyphosphate (IPP), and it is mainly modulated by PHOSPHATE STARVATION RESPONSE 1 (AtPHR1), which is a MYB-CC TF that activates the expression of several genes involved in the transport, scavenging, and recycling of $\mathrm{Pi}[22,23]$. This TF was first characterized in Chlamydomonas reinhardtii under the name of PHOSPHORUS STARVATION RESPONSE 1 (CrPSR1) [24,25]. In A. thaliana, PHR1 controls the transcription of high-affinity Pi transporters to improve nutrient assimilation under Pi deprived conditions. For example, AtPHR1 promotes the expression of PHOSPHATE TRANSPORTER 1 (AtPHT1) to enhance Pi transport across the plasma membrane (PM) [26,27]. AtPHR1 induces the expression of AtPHT2, AtPHT4, AtPHT3, AtVPT1, and AtVPE1 genes, all encoding transporters involved in intracellular Pi redistribution [28-35]. Another aspect of the systemic response controlled by AtPHR1 is the recycling of internal Pi from phospholipids and nucleic acids [36,37]. Pi-deprived plants re-organize the PM composition, replacing membrane phospholipids with non-phosphorus 
lipids such as sulfoquinovosyldiacylglycerol and digalactosyldiacylglycerol [36-38]. Under low Pi conditions, AtPHR1 promotes the transcription of genes involved in lipid turnover such as AtSQD1 and AtPLDz2 [37,38]. AtPHR1 also induces PURPLE ACID PHOSPHATASES (AtPAPs) and RIBONUCLEASES LIKE 1 (AtRNS1) transcription, which are the encoding enzymes that are essential to release Pi from the organic pool $[39,40]$. AtPHR1 activity is negatively regulated by proteins containing the SIG1-PHO81-XPR1 (SPX) domain (encoded by AtSPX1, AtSPX2, AtSPX3, and AtSPX4 genes). The post-translational regulation of AtPHR1 by SPX proteins relies on inositol polyphosphate (IPP) levels [23,41-44]. In high Pi conditions, AtSPX4 binds AtPHR1, retaining it at the cytoplasm [43,44]. At low Pi conditions, AtPHR1 promotes the expression of AtSPX1/2, which is an encoding protein that acts as a repressor of AtPHR1 in the nucleus [23,41,42].

The response of plants to Pi scarcity has been mainly explored using the Arabidopsis root as a model. The root is an essential organ for water and nutrient uptake in diverse clades of the Viridiplantae kingdom; however, we should consider that the root is an innovation during land plant evolution that occurred millions of years later than the developmental and physiological mechanisms that allowed plants to thrive in soils with limited water and nutrients. Pioneer studies on the response of Marchatia polymorpha to Pi limitation described changes in rhizoid abundance, red thallus pigmentation, and the precocious formation of gemmae cups [45-47]. Voth and Hamner (1940) [46] suggested that some segments of thallus tips grow well under low Pi conditions, which may be due to a storage of nutrients which occurred previously facing low Pi conditions. Such studies pointed already to the existence of a molecular program in liverworts to deal with Pi scarcity. However, the molecular and physiological mechanisms of Marchantia to adapt to, and develop in, an environment with low Pi levels remain obscure. In an attempt to shed light on the existence and conservation of such adaptive mechanisms in a rootless early divergent land plant, in this study, we describe the morpho-physiological changes, and the underlying transcriptional profile, of Marchantia polymorpha in response to low Pi availability.

\section{Results}

\subsection{Evolutionary Landscape of Pi Starvation-Induced Genes across the Plant Kingdom}

In the last decades, diverse studies revealed how key genetic networks orchestrate adaptive mechanisms of plants to cope with Pi-limited conditions [7,8]. However, little is known about how these strategies evolved along the early divergent lineages of the plant kingdom. To explore the conservation and divergence of such genetic mechanisms, we performed a survey in key representative genomes distributed across plant phylogeny (Figure 1a). Putative homologs were searched by sequence homology and phylogenetic reconstructions, which allowed us to identify the evolutionary history of genes related to Pi sensing, organic acid exudation, lipid metabolism, and Pi transport (Figure 1a). In the Pi sensing category, putative homologs encoding the internal Pi sensing module PHR1-SPX1 were found in all clades analyzed, from Chlorophyte algae to land plants (Figure 1b). In contrast, in the local Pi sensing genetic network composed of the STOP1, ALMT1, LPR1, PDR2, and RAE1 genes, some members were absent in the Chlorophyte lineage, but each of them was present in all the Streptophyte lineage (Figure 1b). Interestingly, the genome of Ostreococcus lucimarinus lacks four of the five genes related to local sensing, but the other Chlorophyte genomes analyzed (Volvox carteri and Chlamydomonas reinhardtii) possess putative homologs genes of PDR2, LPR1, and RAE1 (Figure 1a). The absence of the STOP1-ALMT1 module in the Chlorophyte sampled genomes was evident, whereas in charophyte algae (Chara Braunii and Klebsormidium nitens), putative homologs of STOP1 and ALMT1 were present (Figure 1a). Our evolutionary analyses highlighted the STOP1-ALMT1 module as a streptophyte innovation. On the other hand, the internal Pi sensing module PHR1-SPX1 was present since early green algae lineages (Figure 1b). Moreover, the putative homologs implicated in lipid turnover, such as XPL, DAG, MGD, DGDG, MGDG, and SQD were highly conserved in all the lineages investigated (Figure 1a). In addition, we explored Pi transport mechanisms and found putative 
homologs of PHT1 and PHF1 in all the species analyzed (Figure 1a), but the putative homologs of NLA were only present in the streptophyte lineage (Figure 1a). We found that PTB genes were present exclusively in both green algae lineages (Chlorophyte and Charophyte) and bryophyte (Figure 1b), as previously reported [48]. Moreover, we found homologs of VPT in all sampled genomes, with the exception of O. lucimarinus (Figure 1a). The last transporter family analyzed was the AtPHO1, and we found putative homologs in all species analyzed in this study (Figure 1a).
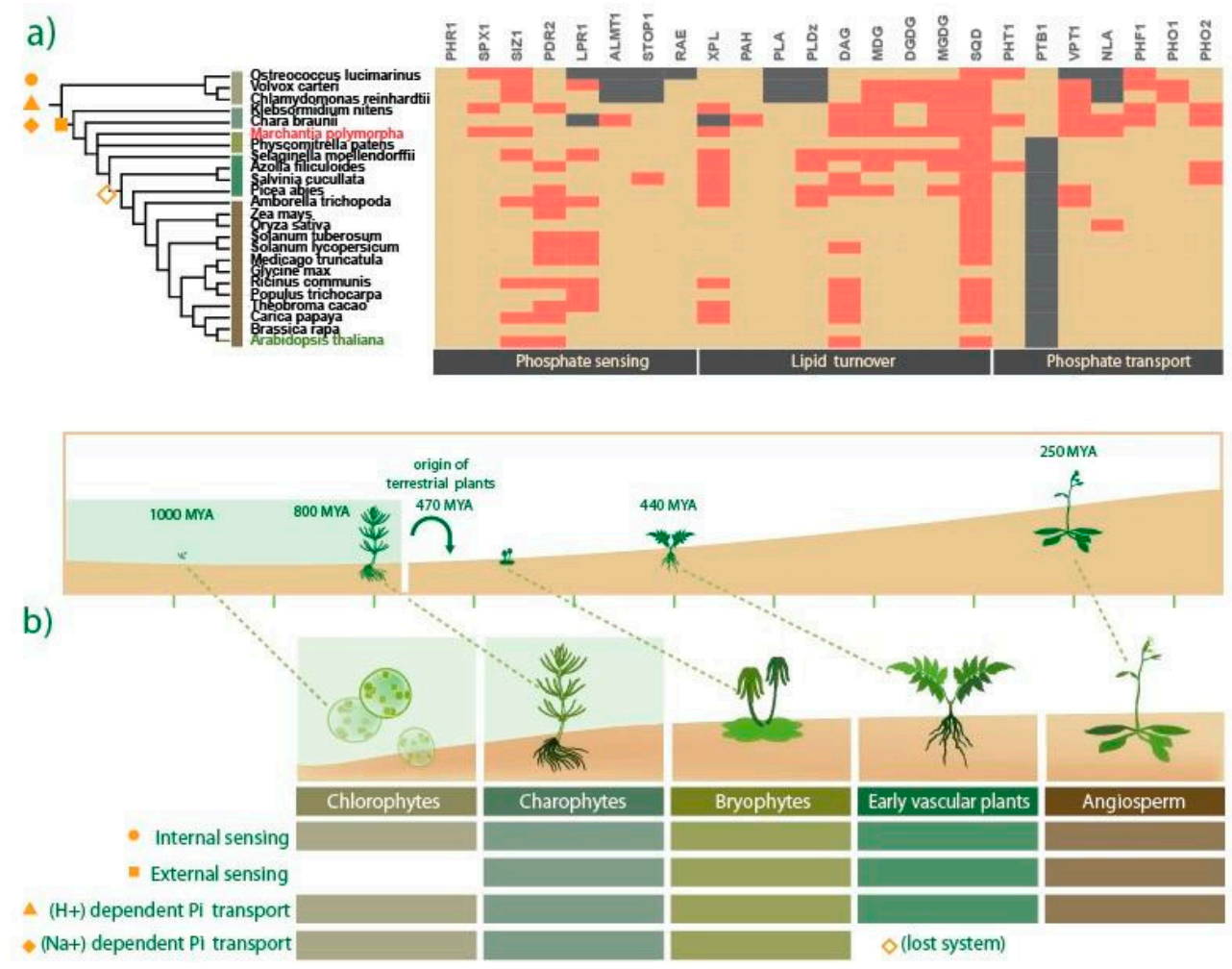

Figure 1. Evolution of phosphate starvation responses in land plants. (a) Phylogenetic tree containing 24 representative genomes sampled across plant kingdom, the cladogram was taken from the NCBI taxonomy browser (https://www.ncbi.nlm.nih.gov/Taxonomy/CommonTree/wwwcmt.cgi). The heatmap shows a comparative conservation analysis of key genes involved in Pi sensing, lipid turnover, and Pi transport. Red-colored rectangles represent single copy genes, beige-colored rectangles mean that for such proteins there are two or more genes, and gray-colored means that no gene coding for such proteins was identified through our approach (see methods for details). Figures S2-S24 show the phylogenetic reconstruction for each gene analyzed. (b) Land plant evolution timeline. A close-up highlight of the morphological differences between chlorophytes, charophytes, bryophytes, gymnosperms, and angiosperms. Color rectangles showed the conservation for key mechanisms to cope with Pi starvation, such as an internal sensing network, external sensing network, $\left(\mathrm{Na}^{+}\right)$and $\left(\mathrm{H}^{+}\right)$dependent Pi transporters.

\subsection{M. polymorpha Exhibits Low Genetic Redundancy in Key Pi-Responsive Regulatory Networks}

M. polymorpha provides a useful model system due to its well-characterized morphology, anatomy complexity [49], and its low genetic redundancy [50]. Our presence-absence analysis of putative homologs related to PSR (Figure 1a) led us to uncover a low genetic redundancy for key genetic networks in M. polymorpha, as compared to other land plants (red rectangles in Figure 1a). For example, the internal Pi sensing module PHR1-SPX1 contains 11 and four members of each family in A. thaliana, respectively. In the M. polymorpha, we identified only three genes encoding putative PHR/PSR-related MYB-CC TFs (Figure 1a). Our phylogenetic reconstruction placed MpPHR1 (Mapoly0003s0147) and MpPHR2 (Mapoly0098s0044) close to AtPHR6, in contrast to MpPHR3 
(Mapoly0115s0048) resides in another subclade with AtPHR8/9/12 (Figure S3). AtPHR1 and AtPHL1 form an independent clade that includes protein sequences from the early vascular plant $S$. moellendorffii up to $A$. thaliana, without bryophyte or green algae sequences. A single gene encoding an SPX-class 1 member was found in M. polymorpha; MpSPX (Mapoly0002s0123) is phylogenetically closest to AtSPX4 (Figure S2). In the lipid turnover category, there are two AtDAG genes, two AtPAH genes, two AtSQD genes, two AtDGDG genes, three AtMGDG genes, three AtMGD genes, and two AtPLDz genes in the $A$. thaliana genome. In the M. polymorpha genome, our analysis retrieved single homologs for $P A H$, $M G D, D G D G, M G D G$, and $S Q D$, (Figure 1a). In the opposite sense, we found nine homologs for $P L D z$ and seven for PLA. Our phylogenetic reconstructions showed that MpPAH (Mapoly0089s0039), MpDAG (Mapoly0022s0098), MpMGD (Mapoly0023s0061), MpMGDG (Mapoly0023s0061), and MpSQD (Mapoly0010s0049) sequences were in a clade with putative orthologs from P. patens and S. moellendorffii (Figures S11, S14, S15, S17 and S18). The phylogenetic tree of DGDG family consists of two clades: one for the $A t D G D G 1$ and the other for $A t D G D G 2$, the latter includes the single MpDGDG sequence (Figure S16). Moreover, we found a single vacuolar Pi transporter in M. polymorpha, MpVPT (Mapoly0094s0020), the A. thaliana genome has three loci encoding for VPTs [35]. Phylogenetic reconstruction of VPT-like proteins showed that MpVPT (Mapoly0094s0020) is in a clade with putative homologs from other bryophytes (Figure S20). Finally, the M. polymorpha genome has a single homolog, MpNLA (Mapoly0044s0127), and A. thaliana possesses two copies where the phylogenetic reconstruction places MpNLA close to other bryophyte sequences (Figure S21).

\subsection{Phenotypical and Physiological Impacts of Pi Availability on Thallus Development}

In natural ecosystems, fluctuations of Pi concentrations in the soil drive fine-tuned mechanisms that allow plants to cope with limiting Pi conditions [7,8]. For example, root morphological modifications and physiological adaptations that respond locally to Pi availability have been associated with enhanced Pi assimilation [51]. However, little is known about the phenotypic plasticity upon Pi starvation in plants without roots, especially in liverworts such as M. polymorpha. To characterize the morpho-physiological responses to low Pi availability during thalli development, gemmae were grown on agar media supplemented with different concentrations of $\operatorname{Pi}(0,10,25,50$, and $500 \mu \mathrm{M})$, and their phenotypes were recorded at multiple time points (7, 14, and 21 days) (Figure S25), allowing us to determine Pi concentrations for high $(+\mathrm{Pi})$ and low $(-\mathrm{Pi})$ growth conditions. The production of a red pigment, most probably auronidin accumulation [52,53], and changes in rhizoid development were observed at 0,10 , and $25 \mu \mathrm{M}$ of Pi (Figure S25). Thus, taking into consideration that thallus development was severely affected at $0 \mu \mathrm{M}$ and $25 \mu \mathrm{M}$ was the highest Pi concentration that induced phenotypic changes, we selected $10 \mu \mathrm{M}$ as a low Pi availability condition. We defined $500 \mu \mathrm{M}$ of Pi as the high availability condition, since at this concentration, thalli neither showed an accumulation of auronidin nor differences in rhizoid development (Figure S25). After registering the alteration on rhizoid development under -Pi conditions, we hypothesized that $M$. polymorpha rhizoids may display similar alterations to those described for $A$. thaliana root hairs under Pi starvation. In order to test such hypotheses, we monitored rhizoid development at each time point of the experiment. Our results showed that at 7 days after sowing (DAS), gemmae developed into young thalli in both +Pi and -Pi conditions (Figure 2a). At this stage, we already observed differences in the length of rhizoids under $-\mathrm{Pi}$ when compared to $+\mathrm{Pi}$ (Figure 2a). The differences between $+\mathrm{Pi}$ and $-\mathrm{Pi}$ conditions were dramatic in both rhizoid length and density at 21 DAS (Figure 2a). In A. thaliana, upon Pi starvation, the increase in root hair density correlates with the excretion of Purple Acid Phosphatases (PAPs) to solubilize Pi from organic compounds [54]. We explored if M. polymorpha rhizoids excrete PAPs by using the phosphatase's substrate EFL-97 or 2-(2'-phosphoryloxyphenyl)-4(3H)-quinazolinone [55], which is converted to $2-\left(2^{\prime}\right.$-hydroxyphenyl $)-4(3 \mathrm{H})$-quinazolinone, a fluorescent product that emits a green signal at $530 \mathrm{~nm}$ wavelength. For this experiment, gemmae were grown under -Pi and + Pi in solid media, both supplemented with $25 \mu \mathrm{M}$ of ELF-97 (see methods for details). After transference to media and being poured into micro chambers to follow each analyzed gemmae, we recorded confocal 
sections at $0,24,48,72,120$, and $168 \mathrm{~h}$ after sown (HAS). Our results are shown in Figure $2 \mathrm{~b}$, where the green signal corresponds to PAP enzymatic activity on ELF-97, and the red signal corresponds to chlorophyll autofluorescence. At 0 HAS, no evident green signal was observed among treatments. By 24 HAS, a discrete green signal was observed in both $+\mathrm{P}$ and -Pi conditions. However, at 48 HAS, a stronger green signal was observed in gemmae grown in -Pi, not only in rhizoid cells but also in surrounding cells (Figure $2 \mathrm{~b}$ magnifications). At this time point, $+\mathrm{Pi}$ grown gemmae also showed green signal in the rhizoids; however, the green fluorescence intensity in these cells was lower, and no evident green signal colocalizing with red signal was observed (Figure $2 \mathrm{~b}$ magnifications). The green signal pattern was even more intense at 72 and 120 HAS under -Pi conditions when compared to those observed in gemmae grown under $+\mathrm{Pi}$ conditions at the same time points (Figure $2 \mathrm{~b}$ magnifications). We also observed in -Pi grown plants that several chlorophyll-producing cells, in the region of the thallus, where rhizoids are formed, also showed a more intense green signal when compared to the control (Figure $2 \mathrm{~b}$ magnifications). Our phenotypic analysis revealed that the proper development and growth of plants, grown under low Pi conditions, was also drastically altered when compared to those of plants grown in $+\mathrm{Pi}$ (Figure 2a). The biomass production was affected lightly after 7 DAS and was clearly lower at 14 and 21 DAS under -Pi conditions relative to the control (Figure 2c). In addition, we observed an evident change in the green color characteristic of the thallus to a strong red/purple color, suggesting that an accumulation of phenylpropanoids (e.g., aunoridins) in response to low Pi conditions is a conserved feature of land plants in response to scarce Pi environments (Figure 2a). The previously described phenotypes correlated with a significant decrease in free Pi in plants grown under low $\mathrm{Pi}$ conditions, in comparison with the control (Figure 2d). Collectively, our phenotypic analyses showed that Pi deficiency causes alterations in early thallus development, some of which were analogous to those previously described for A. thaliana and other land plant species, suggesting that several phenotypic and physiological responses triggered by this stress are conserved across streptophytes.

(a)
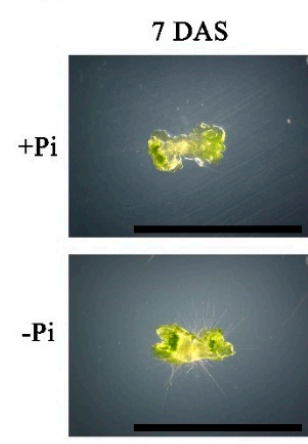

(b)

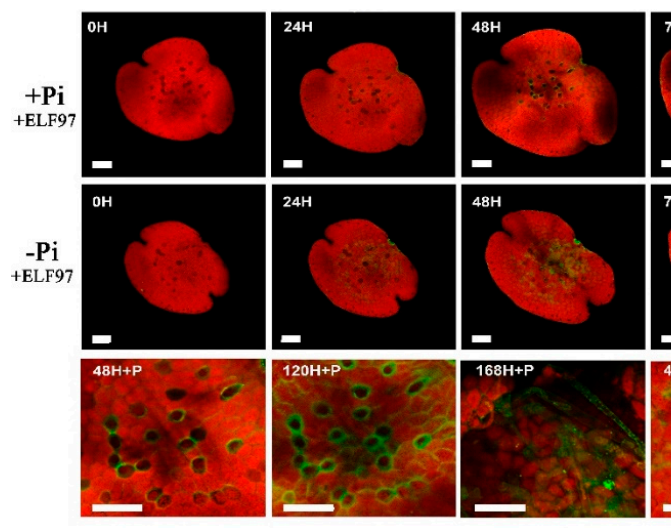

14 DAS
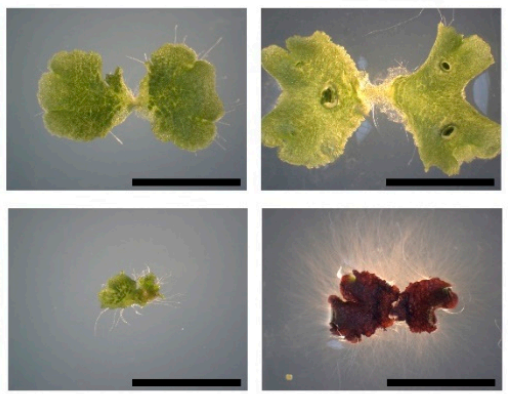

(c)

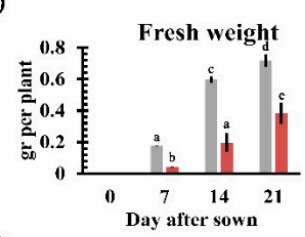

(d)

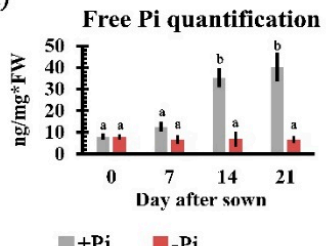

+ $+\mathrm{Pi}-\mathrm{Pi}$

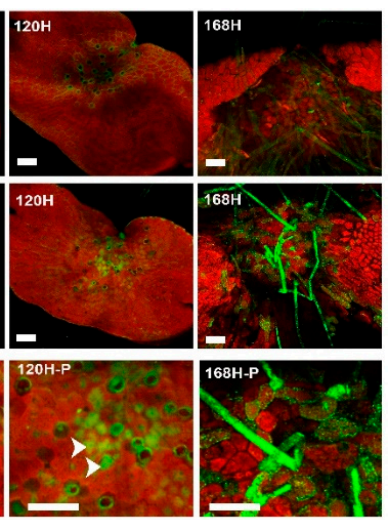

Figure 2. Phenotypical and physiological effect of phosphate availability in Marchantia polymorpha.

(a) Morphological effect of $+\mathrm{Pi}$ and $-\mathrm{Pi}$ status on thalli development in plants at 7, 14, and 21 days after 
sowing (DAS) grown under continuous stressful conditions (black lines $=5 \mathrm{~mm}$ ). (b) In vivo analysis of acid phosphatase activity with ELF-97 in gemmae after 0, 24, 48, 72, and $120 \mathrm{~h}$ after sowing. (c) Fresh weight ( $N=30$ plants) and (d) Free Pi quantification of plants exposed to high and low Pi conditions after 7,14 , and 21 DAS ( $N=30$ plants). The statistical significance was determined with Tukey's honestly significant difference test, letters show significant differences with a $p$ value $<0.01$. All bars in confocal images, panel $(\mathbf{b})=100 \mu \mathrm{m}$.

\subsection{Transcriptional Dynamics in Response to Pi Availability}

The transcriptional response upon Pi starvation has been characterized in diverse angiosperms, such as A. thaliana, Oriza sativa, Zea mays, and other angiosperms, where the transcriptional responses induced by low Pi availability are broadly conserved [56-59]. However, the transcriptional response to low Pi has not been explored in earlier diverging lineages of land plants, such as liverworts. To gain insight into the transcriptional program of young plants relative to Pi availability, we employed an RNA-seq strategy in thalli derived from gemmae 10 DAS (Figure 3a). Plants were grown previously on Pi-sufficient liquid media for 10 days and subsequently transferred to either high $(500 \mu \mathrm{M})$ or low Pi $(10 \mu \mathrm{M})$ MS liquid media (Figure 3a). We defined the times for tissue collection and RNA isolation at 12, 24, and $150 \mathrm{~h}$ post transference (HPT) (Figure 3a), based on the following criteria: (1) the induction of phosphatase activity in low Pi conditions observed at $24 \mathrm{~h}$ after transfer, suggesting early transcriptional activation (Figure 2b); (2) the first distinct phenotypic changes, e.g., rhizoid phosphatase activity, were observed at $168 \mathrm{~h}(7 \mathrm{~d}$ ) after sowing (Figure 2b); (3) the morphological changes in the number and length of rhizoid cells were qualitative evident at $168 \mathrm{~h}(7 \mathrm{~d})$ after sowing (Figure 2a and Figure S25); and (4) the acidification of media, probably by the exudation of organic acids and proton extrusion was observed at 7 DAS in Pi limited conditions (Figure S26). In addition, biological replicates were obtained in an independent experiment under the same growth conditions and RNA collection. Our transcriptome resulted in a total of $274,795,583$ reads, after removing poor quality reads and adapters. To estimate the gene expression profile, we mapped the reads using the DNA subway platform from cyverse (https://dnasubway.cyverse.org/) against the reference genome V3.1 [50]. To determine differentially expressed genes (DEGs), we contrasted libraries from the low Pi conditions against those from plants grown under high Pi conditions. We applied a fold change of $[-1.5<\mathrm{FC}>+1.5]$ and a q-value $<0.001$ as a selection criterion. From a total of 19,138 genes, $6742(35.22 \%)$ were differentially expressed (green bar in Figure 3b), while 12,396 genes did not show significant differential expression (gray bar in Figure 3b). Then, we classified the total DEGs that were up or downregulated at each specific time point (12, 24, and $150 \mathrm{HPT})$. The bar plot in Figure $3 \mathrm{c}$ in orange shows 1463, 1594, and 2258 up-regulated genes. In blue, we found 1202, 1604, and 2570 down-regulated genes at 12, 24, and 150 HPT, respectively. In order to define time-specific and shared DEGs, we performed a Venn diagram analysis for up (orange) and down-regulated genes (blue) (Figure 3d). The up-regulated time-specific DEGs were 599, 625, and 1639 genes at 12, 24, and $150 \mathrm{HPT}$, and the down-regulated time-specific DEGs were 490, 725, and 1825 at 12, 24, and $150 \mathrm{HPT}$, respectively (Figure 3d). In addition, 152 up-regulated genes were shared by the three time points, and 258 down-regulated genes were shared among all times. The remaining DEGs were shared between two time points. For example, in the up-regulated group, samples from 12 and 24 HPT shared 332 DEGs, 24-150 HPT shared 87 and 12-150 HPT shared 380 genes. On the other hand, the down-regulated group showed 289 DEGs shared between 12 and 24 HPT, 322 between 24 and $150 \mathrm{HPT}$, and 165 DEGs shared among 12 and $150 \mathrm{HPT}$ (Figure 3d). In order to reveal the putative functions of DEGs in response to Pi deprivation, we performed a Gene Ontology (GO) enrichment analysis. The total universe of up-regulated DEGs was used to define enriched subcategories from the main category "Biological Processes" $(B P)$ with a threshold of $p$-value $<0.001$. Then, we used the ClueGO software [60] to cluster the over-represented categories in a network, where the circle size correlates to p-value and gray lines define interactions between subcategories (Figure 3e). The major enriched category is the Aromatic acid family metabolic process; it forms a subnetwork including subcategories such as Indole-containing compound metabolic process, Aromatic amino acid family biosynthetic 
process, Cellular amino acid catabolic process, Organic acid metabolic process, and others (Figure 3e). Into these subcategories, we found genes associated with auxin biosynthesis, organic acid cycle, and exudation. Other highly enriched subnetworks include Polysaccharide catabolic process, Starch catabolic process, Glucan catabolic process, and others. Interestingly, the Ion transport category formed another subnetwork, which includes Phosphate ion transport, Iron transport, and other subcategories (Figure 3e). In these subcategories, we found genes involved in Pi and Fe transport, and putative Pi transporter genes such as homologues of PTB and PHT were induced in response to low Pi availability. Another category was the Flavonoid biosynthesis and Chalcone biosynthetic process, where genes related to auronidin biosynthesis were found [52]. The transcriptional activation of these genes correlates with the low Pi phenotype observed (dark purple color on thallus Figure 2a). In addition, we found the conservation of some of the typical responses to low $\mathrm{Pi}$ availability such as the induction of genes underlying the plant hormone genetic networks, organic acid exudation, iron transport, Pi transport, and cellular responses to Pi starvation (Figure 3e). Then, we searched for enriched functional categories of up-regulated genes for each time point; these results are available in the Tables S1-S3. The 599 DEGs specific for 12 HPT (Figure 3d) showed enrichment in 66 functional categories, which include Response to hormones, Auxin polar transport, Response to cytokinin, and the Auxin-activated signaling pathway, among others (Table S1). We found 570 functional categories enriched in the 725 DEGs specific for 24 HPT (Figure 3d). Some of these are related to plant hormones such as Response to abscisic acid and Response to ethylene (Table S2). In addition, we found categories related to processes previously associated with the Pi-local response including Meristem development and Defense response by callose deposition in cell wall (Table S2). The 639 specific DEGS of 150 HPT revealed enrichment in 250 functional subcategories, of which some of the most interesting were Carboxylic acid transport, Citrate metabolic process, Programmed cell death, and Response to aluminum ion (Table S3). Together, our time-specific GO analysis reveals that the transcriptional response of M. polymorpha to cope with Pi starvation is dynamic and involves the up-regulation of genes associated to several key processes, some of which were time-specific and conserved partially with those described in A. thaliana, O. sativa, and Z. mays [56-59].

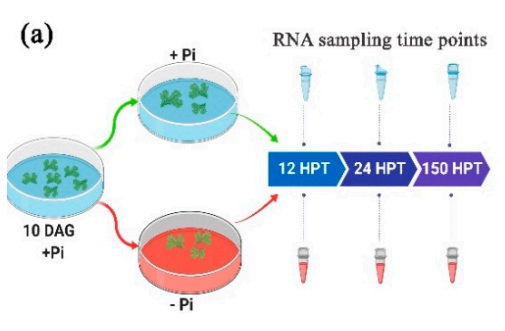

(b)

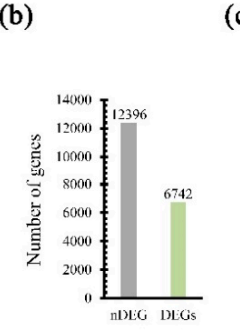

(d)

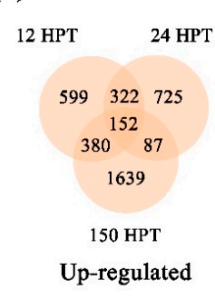

(c) (e)

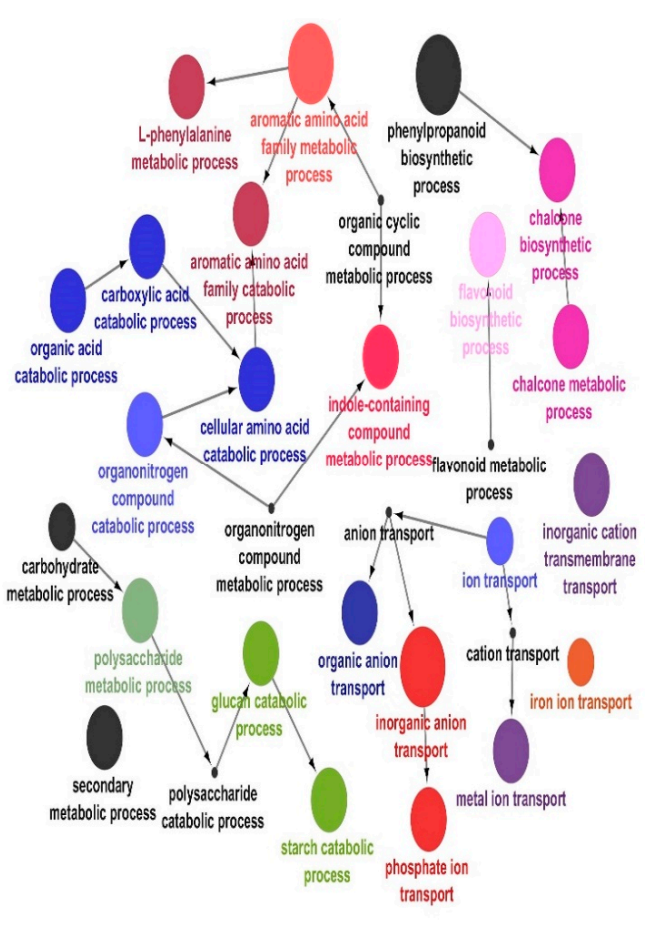

Figure 3. Dynamics of transcriptional responses to Pi starvation. (a) Graphic description of the experimental design for the RNA-seq approach. (b) Differentially expressed genes (DEGs) 
and non-differentially expressed genes (nDEGs). (c) Bar plot showed the up-regulated genes in light red and the down-regulated ones in green for each time of the transcriptome. (d) Venn diagrams for up-regulated (Red) and down-regulated (green) genes for each condition. (e) Clustering of over-represented Gene Ontology (GO) categories in the genes up-regulated in response to low $\mathrm{Pi}$ availability, color clustered similar categories and circle size correlates with the $p$-value of enrichment test.

\subsection{Transcriptional Regulation of the Local and Systemic Pi-Sensing Modules}

In order to explore how Pi sensing occurs in M. polymorpha, we analyzed the transcriptional behavior of genes involved in local and systemic perception networks. Interestingly, only MpPHR1 was up-regulated in low $\mathrm{Pi}$, in contrast to the other two MYB-CC genes (Figure S27). This suggests that MpPHR1 is functionally equivalent to AtPHR1. Moreover, MpSPX was up-regulated across all time points in response to low $\mathrm{Pi}$, similar to that observed for AtSPX1/2. With respect to the local perception network, we found that MpSTOP2 was significantly down-regulated at $12 \mathrm{HPT}$ and up-regulated at 24 and $150 \mathrm{HPT}$, under Pi deficiency. Based on its transcriptional activation, we hypothesized that MpSTOP2 (Mapoly0083s0069) may be functionally equivalent to AtSTOP1. MpALMT3 (Mapoly0100s0061) and MpALMT2 (Mapoly0070s0016) were significantly down-regulated at 12, 24, and $150 \mathrm{HPT}$, even when MpSTOP2 was up-regulated (Figure S27). Although we identified 13 loci encoding multicopper oxidases (MpLPR1 to MpLPR13), only two of them, MpLPR1 (Mapoly0008s0168) and MpLPR3 (Mapoly0008s0270), were up-regulated in all three time points sampled in this work (Figure S27). MpLPR4 (Mapoly0030s0116) and MpLPR7 (Mapoly0186s0019) were up-regulated at 12 HPT and then down-regulated at 24 and 150 HPT. MpLPR11 (Mapoly0217s0007) was up-regulated at 12 and 24 HPT and then down-regulated at 150 HPT (Figure S27). Only MpLPR2 (Mapoly0004s0015) was down-regulated in the three time points analyzed (Figure S27). On the other hand, the CLE peptide family in M. polymorpha contains two members (MpCLE1 and MpCLE2) [61,62]; under low Pi conditions, MpCLE1 (Mapoly1011s0001) was down-regulated at 12 and 24 HPT, and MPCLE2 (Mapoly0084s0052) was up-regulated at 12, 24, and 150 HPT (Figure S27). Taken together, these results indicate that despite the differences, at morphological and genetic levels, with Arabidopsis, M. polymorpha displays a partially conserved transcriptional response of both local and systemic Pi-sensing pathways.

\subsection{Low Pi Promotes the Induction of Genes Related to Organic Acid Synthesis and Exudation}

To unveil if genes involved in organic acid synthesis and exudation were affected in response to low Pi conditions, the transcriptional profiles of key genes such as the malate synthase (MLS), citrate synthase (CIS), malate transporters (ALMT), and citrate transporters (MATE) were analyzed (Figure S28). Our search in the M. polymorpha genome with the Pfam identifiers PF00285 for citrate synthase, PF01274 for Malate synthase, and PF01554 for the MATE transporter revealed five loci encoding putative MALATE SYNTHASE (MpMLS1 to MpMLS5), from which the transcripts of MpMLS2 (Mapoly0016s0095), MpMLS3 (Mapoly0023s0108), and MpMLS5 (Mapoly0154s0035) were down-regulated under -Pi conditions (Figure S28). On the other hand, we found nine loci annotated as CITRATE SYNTHASE (MPCIS1 to MpCIS9); four of them were differentially expressed under low Pi conditions. MpCIS1 (Mapoly0063s0010) was up-regulated at $12 \mathrm{HPT}$ and down-regulated at 24 and 150 HPT, MpCIS2 (Mapoly0071s0012) and MpCIS5 (Mapoly0111s0039) were down-regulated at 12 and 24 HPT, and up-regulated at 150 HPT (Figure S28), and MpCIS6 (Mapoly0200s0007) was down-regulated at the three times analyzed (Figure S28). Regarding malate transporters, MpALMT3 and MpALMT4 were down-regulated, as previously mentioned. This observation correlates with reduced levels of the expression of malate synthesis enzymes and suggests a reduced production and exudation of malate under low Pi conditions. In the case of genes related to citrate transport, we identified 15 loci encoding putative MATE-type transporters (MPMATE1 to MpMATE15), (Figure S28). In Arabidopsis, some members of this family of transporters are involved 
in citrate exudation [63]. Interestingly, MpMATE1 (Mapoly0002s0036), MpMATE3 (Mapoly0005s0170), and MpMATE10 (Mapoly0041s0128) were up-regulated at all the time points sampled (Figure S28). Additionally, another six members of the MATE family were up-regulated in at least one time point sampled (Figure S28). These results suggest that M. polymorpha promotes preferentially the synthesis and efflux of citrate rather than malate to cope with low Pi.

\subsection{Pi Starvation Induces the Expression of Genes Involved in Lipid Turnover}

To explore if phospholipid remodeling takes place in M. polymorpha in response to Pi limited conditions, as previously reported for A. thaliana [36-38], we searched for putative orthologs of key enzymes involved in phospholipid degradation and glycolipid synthesis (Figure 1a). Our results revealed seven loci encoding putative orthologs of type D phospholipases (PLDs), and our phylogenetic reconstruction showed that $M$. polymorpha PLDs formed a basal clade with PLD sequences of bryophytes (Figure S13). Among the M. polymorpha PLD-encoding loci, we found that MpPLD1 (Mapoly0191s0011) is up-regulated at the three time points analyzed and displayed the strongest FC value at $150 \mathrm{HPT}$ (Figure S29). This suggests that MpPLD1 is probably the functional equivalent of AtPLDz2, which is also highly up-regulated in response to Pi deprivation [37]. Although other members of the PLD family, genes in $M$. polymorpha that were down-regulated and up-regulated at different time points showed lower FC values than those of MpPLD1 (Figure S29). In the case of enzymes involved in galacto and sulpholipid synthesis, we found single genes encoding them, MpPAH, MpDGDG, MpDAG, $M p M G D G$, and MpSQD. Among them, MpDGDG and MpMGDG did not show significant changes in their expression relative to the availability of $\mathrm{Pi}$, and $\mathrm{MpDAG}$ was down-regulated at 12 and $24 \mathrm{HPT}$ and up-regulated at $150 \mathrm{HPT}$. In contrast, MpSQD was up-regulated at 12 and $24 \mathrm{HPT}$ and down-regulated at $150 \mathrm{HPT}$ in low Pi (Figure S29). Altogether, these data show that a group of genes, whose homologs in Arabidopsis are involved in lipid turnover, are up-regulated in response to Pi availability in M. polymorpha.

\subsection{Transcriptional Behavior of Genes Involved in ROS Synthesis in Pi-Starved Thallus}

Previous studies have associated reactive oxygen species (ROS), such as hydroxyl radical $\left(\mathrm{OH}^{-}\right)$, superoxide $\left(\mathrm{O}^{-}\right)$, and hydrogen peroxide $\left(\mathrm{H}_{2} \mathrm{O}_{2}\right)$, to the regulation of plant development by acting as secondary messengers [64]. It has been shown in Arabidopsis that NADPH OXIDASE (NOX1) or RESPIRATORY BURST OXIDASE HOMOLOG (AtRBOH), a major enzyme in the production of ROS, is required for the appropriate root hair developmental patterning $[65,66]$. Moreover, transcriptional studies of $A$. thaliana upon Pi starvation revealed that peroxidase-encoding genes were significantly up-regulated [16]. In order to explore the conservation of this response in the early divergent land plant $M$. polymorpha in response to Pi starvation, the transcriptional profiles of $N O X / R B O H$ and peroxidase-encoding genes were analyzed. The Pfam identifiers PF08414 (NADPH oxidase) and PF00141 (Peroxidase) were used to identify the putative M. polymorpha orthologs. We found two loci for NADPH OXIDASE (MpNOX1 and MpNOX2) (Figure S30). The expression of MpNOX1 (Mapoly0258s0001) was up-regulated at 12 and $150 \mathrm{HPT}$, but it was down-regulated at $24 \mathrm{HPT}$ (Figure S30), while MpNOX2 (Mapoly0046s0097) was down-regulated at the three time points sampled (Figure S30). In contrast, we found 221 loci putatively encoding peroxidases (PRX), from which 100 loci showed differential expression in our transcriptomic approach (Figure S30). Transcripts of 24 loci were constantly down-regulated and 14 were up-regulated in the three time points analyzed, and the remaining 62 were up or down-regulated in at least in one of the time points sampled (Figure S30). The transcriptional induction of MpNOX1 under low Pi conditions suggests that M. polymorpha promotes the generation of superoxide radical $\left(\mathrm{O}^{-}\right)$. In addition, the strong induction of several $\mathrm{Mp} P R X$ genes might correlate with ROS production under low Pi conditions and could be involved in the changes observed in rhizoid development upon Pi scarcity. 


\subsection{Validation of Transcriptome by $q P C R$}

In order to evaluate the quality of our transcriptome, seven genes were selected for qPCR analysis. The putative homologs of the selected genes have been associated with relevant aspects of the Pi starvation responses in diverse plant species, such as Pi sensing, cellular response to $\mathrm{Pi}$, organic acid biosynthesis, and transport. Actin was used as control, since it has proven to be an accurate control in previous analyses in diverse stress conditions [67] (Figure 4a). For our qPCR assays, we used cDNA derived from the total RNA sampled at the same time points and growth conditions used for our transcriptome. The transcriptomic profiles of the selected genes are shown in Figure $4 \mathrm{a}$, in which up-regulated genes are depicted in orange, down regulated ones are depicted in blue, and unchanged ones are depicted in black. For the internal Pi perception, our transcriptome showed that MpPHR1 and MpSPX were up-regulated in the three time points analyzed under low Pi conditions (Figure 4a). In contrast, our qPCR analysis showed that MpSPX transcripts under low Pi conditions were less abundant at $12 \mathrm{HPT}$, but they increased drastically at $24 \mathrm{HPT}$ and $150 \mathrm{HPT}$ compared to high Pi conditions as the control (Figure $4 \mathrm{~b}$ ). In the case of MpPHR1, transcript levels increased significantly in response to Pi starvation at the three time points tested (Figure 4e). These qPCR results denote a high coherence with our transcriptomic data for these genes. Regarding organic acid biogenesis, our transcriptomic approach showed that transcript levels of MpMLS3 were down-regulated at all time points sampled and those of MpCIS1 were down-regulated at early times (12 and 24 HPT) and up-regulated at $150 \mathrm{HPT}$ (Figure 4a). The qPCR analysis revealed that transcript levels of MpMLS3 were significantly lower in low Pi at 12 and $150 \mathrm{HPT}$ with respect to the control, but no significant differences between high and low Pi conditions were observed at 24 HPT (Figure 4c). Our qRT-PCR results for MPCIS1 showed no significant differences at early times (12 and 24 HPT), but they highly increased at 150 HPT under low Pi conditions (Figure 4d). Finally, we analyzed the transcriptional behavior of genes whose putative orthologs in A. thaliana are involved in the local perception of $\mathrm{Pi}$ availability. Our transcriptome showed that MpCLE1 was down-regulated at early times (12 and $24 \mathrm{HPT}$ ), whereas the MpCLE2 was up-regulated in the three points sampled, in response to low $\mathrm{Pi}$ (Figure 4a). The qPCR data for MpCLE1 showed low transcript abundance in -Pi conditions at the three time points tested (Figure 4f); on the other hand, transcript levels for MpCLE2 were high under low Pi conditions at the three time points sampled (Figure 4g), showing a high correlation with the expression pattern observed for this gene in our transcriptome approach. In the case of MpLPR1, the transcriptome data showed that the transcription of this gene is not altered between treatments at early times (12 and $24 \mathrm{HPT}$ ) and is up-regulated under low Pi at $150 \mathrm{HPT}$ (Figure 4a). Our qPCR analysis corroborated this expression pattern only after 150 HPT MpLPR1 showed high transcript levels under low Pi conditions (Figure 4h). Overall, our qPCR analysis validated the transcriptional behavior of most of the genes selected, indicating that our transcriptome approach is robust and that the reported expression patterns indeed reflect the response of M. polymorpha to low Pi. 
(a)

\begin{tabular}{|c|c|c|c|}
\hline & $\begin{array}{r}\text { Co } \\
-2-1 \\
\end{array}$ & $\begin{array}{c}\text { lor Key } \\
0 \text { o } 1 \\
02 \text { FC }\end{array}$ & \\
\hline Gene & $12 \mathrm{HPT}$ & $24 \mathrm{HPT}$ & $150 \mathrm{HPT}$ \\
\hline $\operatorname{Mp} L P R I$ & 0 & 0 & 45.78 \\
\hline $\operatorname{Mp} S P X$ & 1.14 & 1.17 & 1.22 \\
\hline $\mathrm{Mp} P H R I$ & 1.24 & 1.54 & 1.07 \\
\hline MpMLS3 & -16.6 & -1.35 & -3.33 \\
\hline $\mathrm{MpCISI}$ & -1.02 & -2.18 & 5.48 \\
\hline $\mathrm{MpCLE1}$ & -3.61 & -1.33 & 0 \\
\hline $\mathrm{MpCLE2}$ & 2.26 & 1.23 & 1.07 \\
\hline
\end{tabular}

(b)

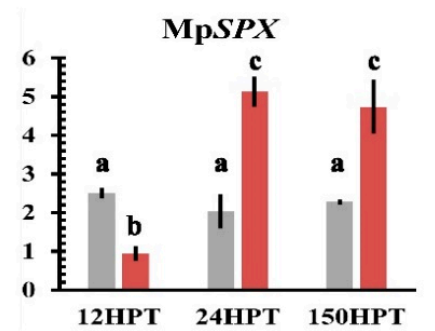

(c)

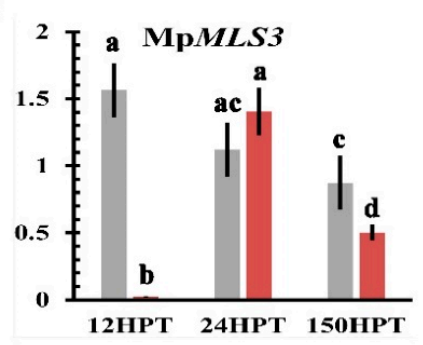

(d)

(e)
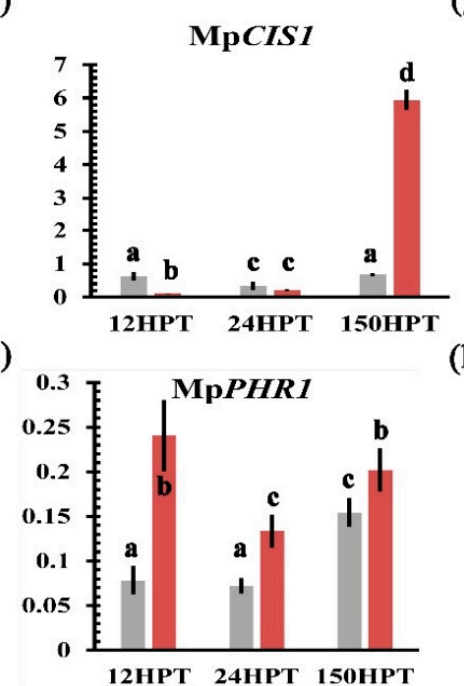

(f)

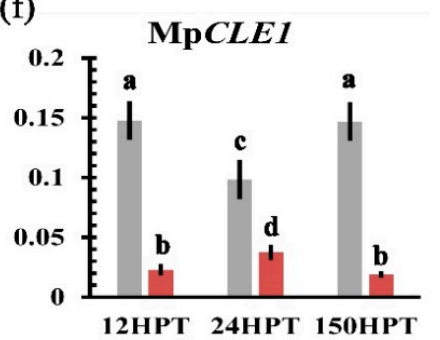

(g)

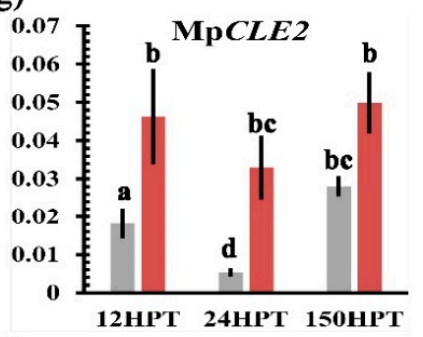

(h)

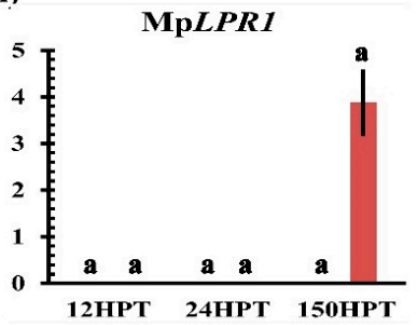

Figure 4. Validation of transcriptome by quantitative real-time qPCR. (a) Heat map showed the transcriptional profile of MpSPX, MpPHR1, MpCLE2, MpLPR1, MpCIS1, MpCLE1, and MpMLS3 resulting from our transcriptome. The gradient of colors represents the following: light blue is down-regulated, black means no change in expression, and orange represents up-regulation. (b-h) Transcript levels of MpSPX, MpPHR1, MpCLE2, MpLPR1, MpCIS1, MpCLE1, and MpMLS genes at 12, 24, and 150 HPT, as determined by qRT-PCR. The Y-axis for all bar plots represents relative expression. The Tukey HSD test was applied for each set of data to find significant differences with an alpha value of 0.05 ; the letters in bars show the relative expression differences in the genes analyzed.

\subsection{Determination of PHR1 Binding Sites (P1BS) Enrichment on DEGs}

Previous studies in A. thaliana reported the enrichment of the PHR1 binding sites (P1BS) cis regulatory elements (CREs) in the promoter regions of AtPHR1 target genes $[13,68]$. Such genes were transcriptionally impaired in the loss of function mutant phr1 and the double mutant phrphl1, unveiling the relevance of the P1BS in the control of Pi starvation responses [68]. We hypothesized that some of the genes that are differentially expressed in response to Pi starvation in M. polymorpha may correlate with the presence of the P1BS motif in their promoter regions. In order to test this idea, we surveyed for the presence and enrichment of the P1BS motif within a $3 \mathrm{~Kb}$ region upstream of the transcription start site of DEGs and non-differentially expressed genes (nDEGs). First, we searched the P1BS consensus sequence (GNATATNC) in the promoters of the 6746 DEGs using HOMER (detailed in Methods) and found that 3259 of them harbor at least one P1BS. Then, we performed a motif enrichment analysis in the promoters of those 3259 DEGs and in the 10,894 nDEGs using the Analysis of Motif Enrichment (AME, Version 5.1.1) [69] tool from the MEME suite [70]. AME identifies if the motif, in this case P1BS, is enriched in a set of sequences considered as primary (DEG and nDEG promoters) compared to a control set of sequences (the $16240 \mathrm{M}$. polymorpha annotated promoter regions). AME scores every sequence in terms of the presence of P1BS and performs a statistical analysis to determine if the primary sequences have better scores than the control sequences. Our results show that 2429 promoters of DEGs are enriched for P1BS, and nDEGs are enriched for 946. Then, promoters from DEGs were 
classified as up-regulated and down-regulated. From the total of up-regulated DEGs (5499 promoters), 3531 have no P1BS, 1188 bear two P1BS, and 780 have one P1BS (Figure 5a). Then, we explored the GO categories of the up-regulated transcripts whose promoters have one and two P1BS, and we found several subcategories highly associated with Pi starvation responses (PSR) such as inorganic phosphate transmembrane transporter activity, triglyceride lipase activity, endoribonuclease activity, and others colored in red in the network (Figure 5b). The subcategories not related directly to PSR appear colored in black (Figure 5b). In the case of down-regulated DEGs, we analyzed a total of 1247 promoter regions for enrichment in the P1BS CRE and found that 142 have one P1BS, 319 have two P1BS, and 786 have no P1BS (Figure 5c). Respective GO analysis for those that contain the P1BS motif revealed subcategories that are linked to the pathogen response, i.e., endopeptidase inhibitor type $C$, in blue [71], and other subcategories are colored in black (Figure $5 \mathrm{~d}$ ). The last group contained genes whose expression did not show significant changes (10894 nDEGs), and we found that only 500 and 546 promoters have one and two P1BS, respectively (Figure 5e). In contrast, the remaining 9803 have no P1BS. The GO analysis of this group revealed categories that in Arabidopsis have been related to Pi starvation (in red) and stress responses in others that have been previously related to AtPHR1, i.e., Zinc ion binding and inositol monophosphate phosphatase activity [71,72] (Figure 5f). Overall, these results show that the number of promoters enriched for P1BS is higher in up-regulated DEGs than in nDEGs. However, the up-regulated and down-regulated promoter groups displayed similar tendencies for P1BS enrichment, but the GO analysis revealed that within the up-regulated group, we mainly found subcategories related to PSR. By contrast, in the down-regulated targets, we found an overrepresentation of subcategories related to the response to pathogens. Hence, the presence of P1BS in the down-regulated and nDEGs suggests that MpPHR1 could be involved in the transcriptional regulation of diverse targets involved in a broad range of stress conditions [71-73].

(a)

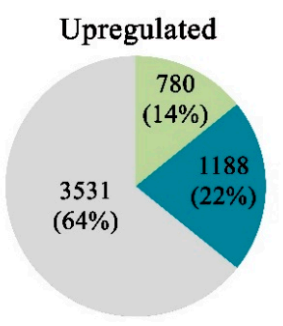

(c)

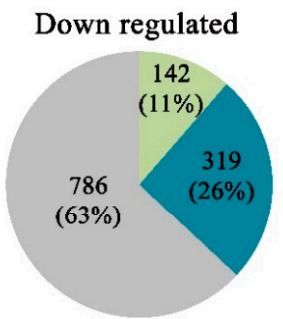

(e)

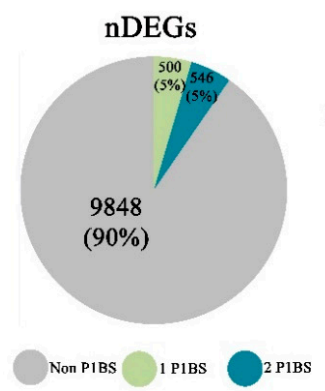

(b)

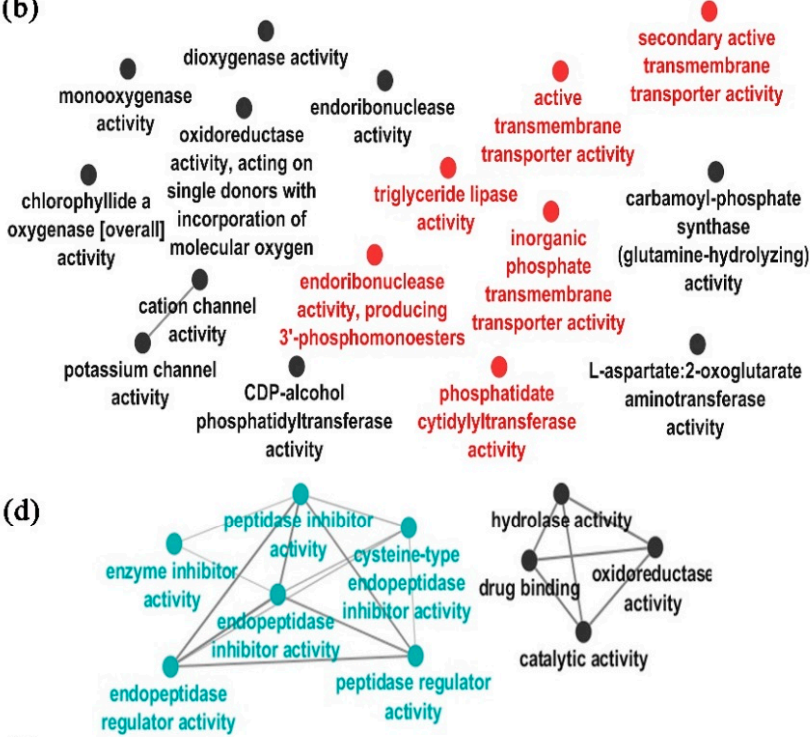

(f)

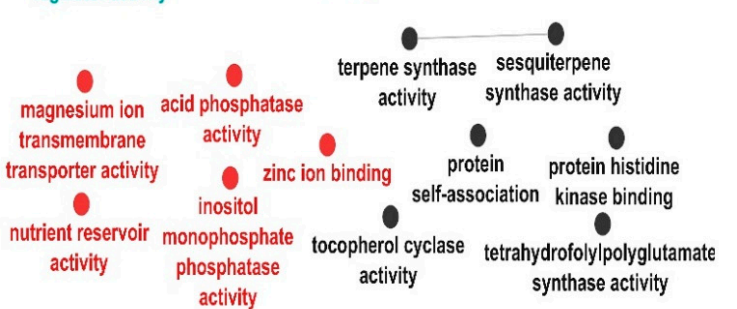

Figure 5. Genome-wide identification of PHR1 binding sites (P1BS) distribution on M. polymorpha. (a) Pie chart showing the number of promoter regions of up-regulated DEGs that lack the P1BS motif (in gray) and those whose promoter has one (green) or two (blue) motifs; the same color code applies 
for those pie charts y (c) and (e). (b) Illustrates the GO analysis of the up-regulated DEGs that contain the P1BS CREs; red color indicates the subcategories that are strongly associated to Pi starvation responses, and black indicates those terms that have no evident link to the Pi starvation responses (PSR). (c) Pie chart showing the number of promoter regions of down-regulated DEGs that lack the P1BS motif and those whose promoter has one or two motifs. (d) The GO network for the down-regulated genes with P1BS motifs, blue nodes indicate subcategories that have been related to PHR1 function in regulating genes involved in other stress conditions described in A. thaliana [71], dark nodes show categories with no evident link to the Pi starvation response. (e) Pie chart showing the number of promoter regions of non-DEGs that lack the P1BS motif and those whose promoter has one or two motifs. (f) The GO networks found after analysis of the genes with P1BS motives in the nDEGs group; subcategories related to low Pi availability appear in red, whereas black indicates the subcategories non-associated to the Pi limitation response.

\section{Discussion}

\subsection{Morpho-Physiological Responses upon Pi Starvation}

In the present work, the morphological and physiological responses of the rootless plant M. polymorpha to low Pi availability were described. Among these responses, we found an increase in the size and number of rhizoids under low Pi conditions (Figure 6). In A. thaliana, the increase in root hair density during Pi deprivation has been described as a strategy to improve the assimilation zone in the rhizosphere [51]. Hence, the modulation of rhizoid development under limited conditions of Pi could be involved in enhancing Pi uptake. It has been shown in M. polymorpha that rhizoid development is regulated by auxin signaling and metabolism [73-76]. We also found that a biomass accumulation of thalli was negatively affected under low Pi conditions, as shown in Figure 6. This phenotypic change, as a function of Pi availability, has been also observed in other plant models such as in Arabidopsis thaliana, Oryza sativa, and Zea mays $[35,43,58]$. We also demonstrate that free Pi levels are lower in plants grown under -Pi conditions compared to those grown in +Pi conditions, suggesting that the decrease of internal Pi amounts in M. polymorpha may trigger the expression of MpPHR1 targets, as reported in A. thaliana (Figure 6). In A. thaliana, the transcriptional activation of AtPHR1, under low Pi conditions, is controlled by AtARF7 and AtARF19 through three auxin response elements found in its promoter [77], suggesting that MpARFs may regulate MpPHR1. However, future experiments are necessary to test this hypothesis. After 21 days of growth under low Pi, thalli exhibited an intense red/purple color, denoting an accumulation of phenylpropanoids (i.e., auronidins in the case of M. polymorpha), which is a common phenotype associated with Pi deficiency across land plants. In A. thaliana, the activity of key genes such as the PHENYL AMONIOLIASE (AtPAL) and CHALCONE SYNTHASE (AtCHS), which are both involved in anthocyanin biosynthesis, were up-regulated under low Pi conditions [56]. Our GO analysis of up-regulated DEGs revealed enriched functional categories related to Flavonoid biosynthesis and Chalcone metabolic processes, which are both related to auronodin biosynthesis. The biochemical features of auronidin have been reported early, as well as the effects of UV light [78] and nutrient deprivation [52,79] in triggering its synthesis [53]. Here, we report changes in the transcriptional patterns on key biosynthetic genes such as chalcone synthase, phenyl amonieliase, and the transcription factor MpMYB14 (Mapoly0073s0038) mainly at 150 HPT (Figure S31). Another important aspect of the Pi-starvation response reported in vascular plants is the secretion of PAPs and OA to the rhizosphere. Our in vivo analysis of phosphatase activity revealed a strong induction of PAP's activity in response to limited Pi, as previously reported for Z. mays, O. sativa, and C. reinhardtii [71-82]. Our results suggest a partial conservation in $M$. polymorpha in the induced OA production, while the transcriptional evidence suggests a preference in the synthesis and exudation of citrate rather than malate. 


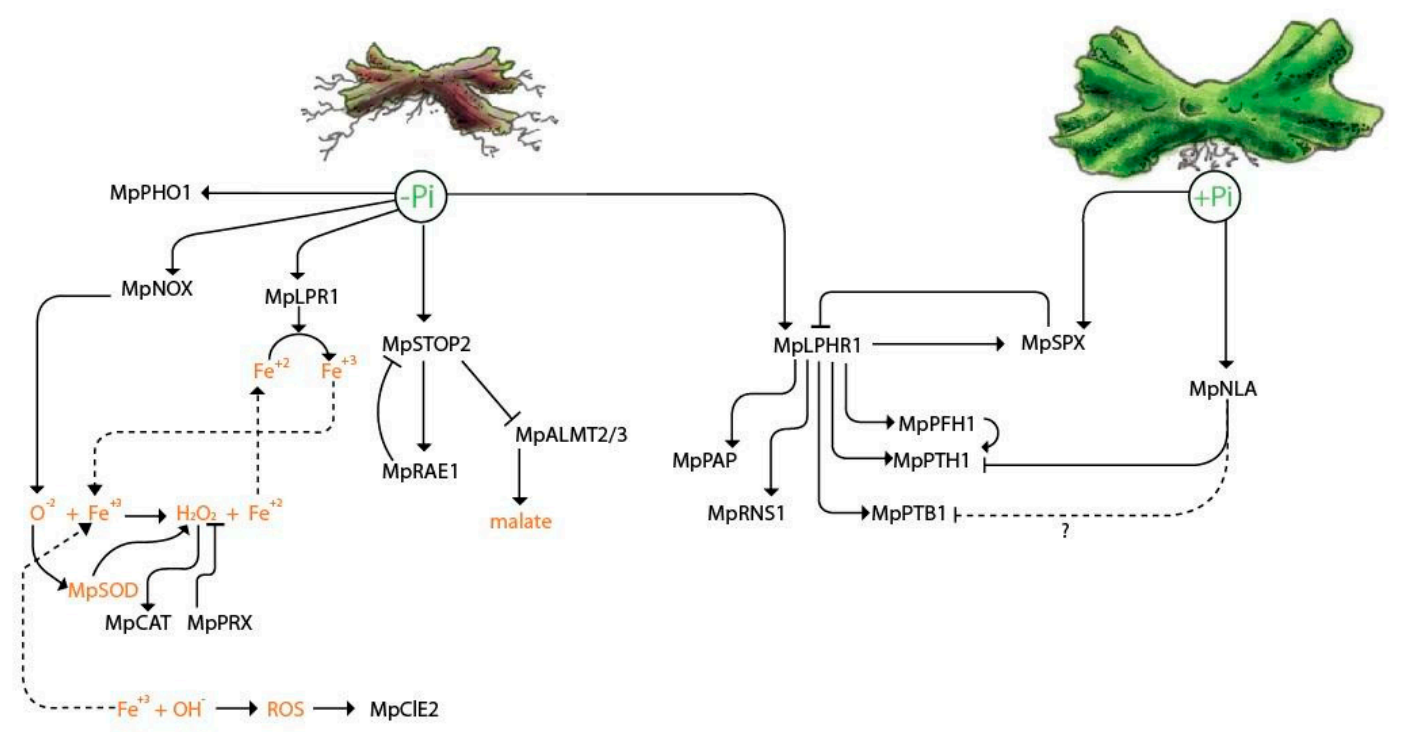

Figure 6. Model of the molecular response of M. polymorpha upon Pi starvation. Under low Pi conditions (left side of the figure), the expression of MpPHO1 is promoted. MpNOX1 induces the production of superoxide radical $\left(\mathrm{O}^{2-}\right)$, which is catalyzed by SUPEROXIDE DISMUTASE (MpSOD) into hydrogen peroxide $\left(\mathrm{H}_{2} \mathrm{O}_{2}\right)$. Hydrogen peroxide is catalyzed by CATALASE (MpCAT) into oxygen and water. In addition, the peroxidases $(\mathrm{MpPRX})$ use hydrogen peroxide to carry out several reactions. On the other hand, the $\left(\mathrm{O}^{2-}\right)$ plus $\mathrm{Fe}^{3+}$ carried out the Fenton-Weiss reaction to produce hydroxyl radical and $\mathrm{Fe}^{2+}$. This step coupled to the induction of MpLPR1, which is a multicopper oxidase that leads to $\mathrm{Fe}^{3+}$ from $\mathrm{Fe}^{2+}$ under low Pi conditions, generates an oxidation-reduction process, and probably impacts thallus development similar to what occurs in A. thaliana root. Here, we observed that the gene encoding the MpCLE2 peptide is up-regulated in specific times after growth in low Pi conditions-probably to modulate the maintenance and function of the meristem. In addition, during low Pi deficiency, the expression of MpSTOP2 is up-regulated, promoting the expression of

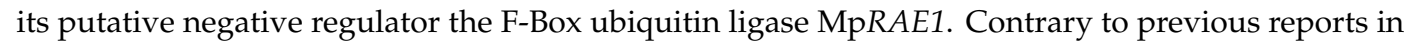
vascular plants, MpALMT2/3 are down-regulated under low Pi conditions. In agreement with this, we hypothesized that low Pi represses the expression of malate transporters to reduce the efflux on soil. On the other hand, the transcription factor MpPHR1 is induced under low Pi conditions and promotes the expression of MpPAPs and MpRNS1, which are both genes involved in Pi scavenging. MpPHR1 promotes the expression of both Pi transporters MpPHTs and MpPTBs via the P1BS motives in their promoters. Additionally, we found that the MpPHF1, an essential protein for the movement of MpPHT transporters from the ER to the PM, is under the control of MpPHR1. In contrast, MpNLA is expressed in high Pi conditions, leading to the degradation of PHT and probably the degradation of the MpPTB transporters. Finally, among the MpPHR1 targets induced during Pi limitation, we found MpSPX, which is a negative regulator of MpPHR1. MpSPX is up-regulated under low Pi conditions under the control of MpPHR1, and another mechanism leads to its expression on high Pi availability.

\subsection{Evolutionary Perspective of Pi Starvation Responses along Viridiplantae}

Pi sensing capacity is one of the major tools of plants to cope with Pi scarcity. In A. thaliana, two independent networks involved in the perception of internal and external Pi levels have been described. Our evolutionary analysis unveiled a high conservation of the PHR1-SPX1 module for the internal Pi sensing networks in M. polymorpha, and we found that putative homologs have been conserved since chlorophyte algae (Figure 1a; Figure 6). However, the functional conservation of the PHR1-SPX module in green algae and M. polymorpha remains pendant. On the other hand, we found that the STOP1-ALMT1 regulatory network is a key innovation of streptophyte lineages (Figure 1a; Figure 6). Therefore, we speculate that the STOP1-ALMT1 network was an innovation that influenced OA efflux and modified earth crust during the silicate weathering period, perhaps helping to reduce the 
levels of atmospheric $\mathrm{CO}_{2}$ [83]. The replacement of phospholipids by glycolipids in the lipidic bilayer of cell membranes is a well-described mechanism to deal with Pi scarcity, which is highly conserved, even in bacteria [84]. We found a strong conservation of XPL, PHA, DAG, MDG, DGDG, MGDG, and $S Q D$ putative orthologous genes from chlorophyte algae, streptophyte algae, and M. polymorpha. However, genes encoding PHOSPHOLIPASE alpha (PLA) and PHOSPHOLIPASE D (PLD) were not found in the chlorophyte genomes analyzed. We hypothesized that other phospholipases, such as PHOSPHOLIPASE C (PLC-L) and PHOSPHOLIPASE B (PLB), could be converting phospholipids into DAG for the production of sulfo and galactolipids, as it was reported for $C$. reinhardtii under nitrogen limitation [85]. Other key genes that we found to be highly conserved are the putative PHT1 transporters, which are found along plant phylogeny from chlorophyte algae to vascular plants (Figure 1a). However, we found that sodium-dependent Pi transporters (PTB) are present only in green algae and M. polymorpha, suggesting that vascular plants lost this type of transporters. In agreement with these findings, the conservation of PTB transporters in M. polymorpha was reported in a previous study [48]. In addition, VPT and PHO1 orthologous genes were found highly conserved from green algae to land plants. NLA and PHF genes, involved in the regulation of PHT transporters, were present only in the streptophyte lineages (Figure 1a). The establishment of this regulatory mechanism could be linked to the process of land colonization by plants as a possible innovation in the regulation of $\mathrm{Pi}$ uptake in terrestrial environments. Probably, this innovation occurred to avoid the Pi toxicity in soil, since the concentration of available Pi on land is higher than in aquatic environments.

\subsection{Transcriptional Response to Cope with Limited Pi in M. polymorpha}

Our work opens several working hypotheses, which include one very interesting gene: MpNOX1, which is partially up-regulated under low Pi conditions, suggesting that Pi limitation promotes superoxide radical production via $M p N O X 1$, connecting ROS signaling to morphological responses (Figure 6), probably to modulate rhizoid development, similarly to what occurs in $A$. thaliana root hairs [65]. Other interesting genes identified were those encoding CLE peptides; the expression of MPCLE2 was up-regulated, whereas MpCLE1 was down-regulated under low Pi (Figure 4; Figure 6). Previous work showed that the ectopic expression of MPCLE1 reduces the thallus growth area of Marchantia plants grown in sufficient Pi conditions; on the other hand, the loss of function mpcle1 mutant [61] alters the thallus proliferative region and affects the organization of dividing cells. On the other hand, exogenous application of the MpCLE2 peptide alters the differentiation of stem cell-derived daughters, causing the accumulation of the stem cell pool which, after MpCLE2 removal, leads to dichotomous branching [62]. Our transcriptional analysis showed that MPCLE2 levels are induced upon Pi starvation, and those of MpCLE1 are repressed. We hypothesized that under low Pi conditions, the altered expression of both MPCLE1 and MPCLE2 may be affecting the proper organization and maintenance of the meristem, which could be reflected in the aberrant development of the thalli observed in plants growing for prolonged time under Pi conditions. Future studies including time-lapse experiments of reporter lines for both MpCLE genes will be very useful to explore this hypothesis.

Our study describes the transcriptional response of M. polymorpha upon Pi starvation and integrates a genome-wide search for the P1BS motive enrichment. Our results show a clear enrichment in P1BS motifs in promoters of DEGs as compared to the nDEGs universe (Figure $5 \mathrm{a}, \mathrm{c}, \mathrm{e}$ ). The similar enrichment observed in both up-regulated and down-regulated genes suggest that MpPHR1 or other MYB-CC TFs could participate in modulating the expression of genes involved in other processes. This hypothesis is in agreement with early reports in A. thaliana, where AtPHR1 was shown to be involved in the regulation of genes related to zinc and iron homeostasis [71,73]. Moreover, AtPHR1 controls the expression of some genes involved in the plant immune response, indicating that the genetic network controlled by AtPHR1 is highly complex and responds to several environmental cues [71]. Despite the number of up-regulated DEGs being higher than that of down-regulated DEGs, we speculate on why a gene that has the P1BS motif in its promoter is down-regulated in response to Pi starvation. One possibility is that if we consider that MpPHR1 and MpPHR-like TFs are all able to bind the P1BS 
motif, some of the members of the family may act as repressors over a subset of targets. This type of opposite action of diverse members of a TF family has been shown in M. polymorpha for MpARFs $[75,86]$. Future studies should explore such a scenario, since the GO analyses of the down-regulated DEGs, containing the P1BS, revealed interesting functional categories such as Cysteine type endopeptidase inhibitor, which contain genes in which the homologs in other species are expressed in response to diverse plant pathogen and some components of the plant immune system [71,87]. This suggests that M. polymorpha might also be prioritizing the nutrition mechanisms over the pathogen defense response, as it occurs in other plant species. Such defense plasticity could allow the interaction with beneficial organisms [71]. Future studies should include the generation loss of function mutants of MpPHR1 and MpPHR-like genes in order to know the direct targets for each of them, as well as to explore their regulatory roles in response not only to low Pi but also upon other biotic and abiotic stresses. Collectively, our morphophysiological, transcriptional, and promoter analyses for P1BS revealed a core of partially conserved responses deployed to cope with low Pi availability in M. polymorpha, which were analogous to those displayed by $A$. thaliana. We consider that such conserved adaptations, and the M. polymorpha specific traits, should be revised and experimentally tested, case by case, in order to characterize in detail the global strategy of this liverwort to deal with Pi scarce environments.

\section{Materials and Methods}

\subsection{Evolutionary Analysis}

In this work, we searched for classical genes related to phosphate sensing, root system architecture modification, organic acid exudation, and phospholipid turnover along the viridiplantae phylogeny. We selected, as the query, previously characterized Arabidopsis genes involved in the response $[7,8]$. To identify the orthologous genes in M. polymorpha and other 23 representative genomes along the Viridiplantae phylogeny (Figure S1), we performed a search by sequence homology with the software blast v.3.1 [88] used as a threshold parameter an e-value 1e-30 for land plants. For green algae genomes, we used an e-value 1e-10. Additionally, reciprocal blasts against the Arabidopsis protein database TAIR.ver.10 under the same threshold conditions were carried out. The multiple sequence alignment (MSA) was generated with MAFFT software version 7, on the online website [89]. Afterwards, the MSA was trimmed using TrimAL software version 3, maintaining at least $40 \%$ for each original protein alignment [90]. Then, we selected the best model fit to carry out the phylogenetic inference. The ModelTest software was used to analyze the sequences according to ACR criteria [91]. For the phylogenetic reconstruction, we used RAxML software on the CIPRES environment (https://www.phylo.org/portal2/home.action) [92]. The bootstrapping value used for each reconstruction was 1000, and the best tree was plotted using iTOL (https:/itol.embl.de/) [93].

\subsection{Plant Material and Growth Conditions}

Gemmaelings from M. polymorpha (Tak-1) were sown under Murashige and Skoog (MS) media (1/10), pH 5.5, $1 \%$ of sucrose $(w / v)$ and agar $10 \mathrm{~g} / \mathrm{L}$. Plants were grown in Percival chambers with continuous light, with a temperature of $22{ }^{\circ} \mathrm{C}$ and humidity around to $60 \%$. To determine the Pi concentration for high and low availability, gemmaelings were exposed to a gradient of K2PO4 $(0,10$, 25, 50, 100 and $500 \mu \mathrm{M}$ ) for 21 days. Phenotypic effects were followed and photographed, using a Zeiss stereoscope, at 7, 14, and 21 days after sown (DAS). For further experiments, we used for low $\mathrm{Pi}(-\mathrm{Pi})$ a $10 \mu \mathrm{M}$ concentration and for high $\mathrm{Pi}(+\mathrm{Pi})$ a $500 \mu \mathrm{M}$ concentration of K2PO4. In addition, for RNAseq experiments, we use MS (1/10) liquid media, $\mathrm{pH} 5.5,1 \%$ of Sucrose $(w / v)$, supplemented with $\mathrm{K} 2 \mathrm{PO} 4$ to establish the $+\mathrm{Pi}$ and -Pi conditions. The implementation of liquid media for RNAseq sample preparation was due to in solid media thallus-carried agar traces in the rhizoids. The plants grow in $+\mathrm{Pi}$ conditions for 10 days under continuous light, $22{ }^{\circ} \mathrm{C}$, and humidity around to $60 \%$. Then, we washed the thallus with distilled water and transferred it to +Pi and -Pi media. After 12, 24, and $150 \mathrm{~h}$, the tissue was collected for RNA extraction. 


\subsection{In Vivo Phosphatase Activity Assay}

The gemmae from M. polymorpha (Tak1) were sown under MS media (1/10) supplemented with $1 \%$ of sucrose $(w / v)$, phytagel $3 \mathrm{~g} / \mathrm{L}, \mathrm{pH} 5.5$ and displayed on polycarbonate cell growth chambers. In addition, the $\mathrm{Pi}(\mathrm{KH} 2 \mathrm{PO} 4)$ was supplied to establish the $+\mathrm{Pi}$ and $-\mathrm{Pi}$ conditions, respectively. To detect the phosphatase activity, we added after sterilization ELF-97 reagent to a final concentration of $50 \mu \mathrm{M}$. Plants were grown in Percival chambers with continuous light, temperature $22{ }^{\circ} \mathrm{C}$, and humidity around to $60 \%$ for 5 days. For phosphatase activity, we recorded gemmae development growth in high and low Pi conditions in the presence of ELF97. Confocal images were recorded at diverse time points by using CLSM Zeiss LM800 microscope, chlorophyll auto-florescence was observed at a $610 \mathrm{~nm}$ wavelength and ELF-97 was observed at $530 \mathrm{~nm}$.

\subsection{Free Phosphate Quantification}

Free Pi was quantified by the malachite green assay [27], modified, and adapted measurements in young thalli. First, we ground the fresh samples $(50 \mathrm{mg})$ in liquid nitrogen, added $500 \mu \mathrm{L}$ of MES buffer $(0.17 \mathrm{M}, 100 \mathrm{mM}$ DTT, $\mathrm{pH} 5.8)$ to each sample, and mixed vigorously. After centrifugation at 12,000 rpm for $10 \mathrm{~min}$, the supernatant was collected in a new tube. Then, $50 \mu \mathrm{L}$ of each sample were mixed with $115 \mu \mathrm{L}$ of acid molybdate solution (17.55 g/L of ammonium molybdate tetrahydrate, $3 \mathrm{M}$ sulfuric acid) and $700 \mu \mathrm{L}$ of deionized water. Ten minutes later, $150 \mu \mathrm{L}$ of green malachite solution (3.5 g/L polyvinyl alcohol, $0.35 \mathrm{~g} / \mathrm{L}$ malachite green) were added. The absorbance was read at $610 \mathrm{~nm}$ after $2 \mathrm{~h}$. A titration curve was performed in the same conditions, using a stock solution $1 \mathrm{mM} \mathrm{KH} 2 \mathrm{PO} 4$ to measure 25 , $12.5,6.25,3.13,1.56$, and $0 \mu \mathrm{M}$, respectively. Finally, to calculate the Pi concentration in each sample, we used a regression equation normalized with the respective data from fresh weight.

\subsection{RNA Extraction, Sequencing, and Quantitative Real-Time qPCR Analysis}

For RNA-seq, samples for RNA extraction (from two independent biological replicates per each condition and time point) were collected and ground in liquid nitrogen. For each sample, $900 \mu \mathrm{L}$ of TRIzol reagent were added and incubated in ice for several minutes and $5 \mathrm{~min}$ at room temperature (RT). Previous to centrifugation at $13,000 \mathrm{rpm}$ for $10 \mathrm{~min}$ at $4{ }^{\circ} \mathrm{C}$, we add $200 \mu \mathrm{L}$ of chloroform/isoamyl alcohol (24:1) and mixed briefly for $2 \mathrm{~min}$ at RT. The supernatant was collected in a new tube and incubated for $10 \mathrm{~min}$ at RT with $500 \mu \mathrm{L}$ of isopropyl alcohol stored previously at $-20^{\circ} \mathrm{C}$; later, it was precipitated by centrifugation at $13,000 \mathrm{rpm}$ for $10 \mathrm{~min}$ at $4{ }^{\circ} \mathrm{C}$. Finally, the precipitated RNA was washed with ethanol $75 \%(v / v)$, stored at $-20^{\circ} \mathrm{C}$, and was resuspended in DEPC water. Before sequencing, the RNA samples were analyzed with Bioanalizer, and RIN values lower than 8 were discharged. Libraries were prepared using TruSeq RNA and sequenced with ILLUMINA next seq 1x75 for each replicate, time, and condition. For qPCR experiments, the total RNA was extracted and analyzed as described above. We used $5 \mu \mathrm{g}$ of total RNA for each $20 \mu \mathrm{L}$ reaction to obtain cDNA for each condition and time. cDNA was synthesized using SuperScript II (Invitrogen, Carlsbad, California, USA) according to the manufacturer's protocol. The expression quantification was determined using SYBR Green PCR master mix (Applied Biosystems, MA, USA) in $10 \mu \mathrm{L}$ reaction. The levels for each tested transcript were determined using three technical replicates per biological replicate and normalized to actin transcript levels. The results are represented as $\left[2^{(-\Delta C t)}\right]$, the primers used in these reactions are listed in Figure S32.

\subsection{Differential Expressed Genes Determination and GO Enrichment Analysis}

The RNAseq dataset is available on the NCBI repository under the bioproject identifier PRJNA667150. We collected raw data to perform quality analysis with the software FastQC. In addition, the reads were processed with Trimmomatic software to remove the adapter contamination [94]. Then, we uploaded the filtered data to the Cyverse environment (https://de.cyverse.org/de/) and use the DNA subway tool (https://nnasubway.cyverse.org/) to generate RNAseq analysis to obtain the 
differentially expressed genes. We used the pipeline established for the tuxedo suit, the DEGs were selected based on FC $(>1.5$ and $<-1.5)$ and FDR $<0.001$. Venn diagrams were performed using the online tool (http://bioinformatics.psb.ugent.be/webtools/Venn/). On the other hand, the enrichment analysis of Gene Ontology (GO) terms was carried out for the DEG selected each time. The GO analysis was performed in the online tool PlantRegMap (http://plantregmap.gao-lab.org/go.php); we selected the M. polymorpha genome to perform the analysis of biological processes categories, and the $p$-value was lower than 0.001 .

\subsection{P1BS Search in DEGs}

To identify the PHR1 DNA binding site (P1BS) in the cis-regulatory region of differentially expressed genes, we first retrieved a $3 \mathrm{~kb}$ fragment upstream of the start codon of all M. polymorpha genes detected in our transcriptomes using the v3.1 genome release available in Phytozome 13 (https://phytozome-next.jgi.doe.gov/). Then, we searched for the presence of P1BS in these cis-regulatory regions using HOMER (v4.11, 10-24-2019) [95]. For this, we used the P1BS consensus sequence (GNATATNC) to make a motif file with the seq2profile.pl tool, and then we queried the cis-regulatory regions of all the differentially expressed genes with the findMotifs.pl tool. The upstream regions of non-differentially expressed genes were selected as background for this analysis. Once we had the P1BS occurrences and their sequence, we built a sequence logo that represented the P1BS in M. polymorpha with ggseqlogo [96] in R.

\subsection{Analysis of P1BS Enrichment}

To determine if the P1BS was relatively enriched in the promoter set of differentially expressed genes compared with the promoter set of all the genes in our transcriptome, we used AME [68], a tool from the MEME suite [69]. We provided AME with the P1BS motif sequence to search and did two analyses in which we wanted to test for P1BS enrichment: the set of 3259 promoters with at least 1 P1BS obtained from the initial P1BS search with HOMER, and the 10,783 promoters belonging to non-differentially expressed genes. For both analyses, we utilized the 16,240 promoters available in the Marchantia polymorpha v3.1 genome release (https://phytozome.jgi.doe.gov) as a control sequence set. We selected an average odds score as a sequence scoring method and the Fisher's exact test as the motif enrichment test. The list of promoters with P1BS enrichment is available on the Table S4.

Supplementary Materials: The following are available online at http://www.mdpi.com/1422-0067/21/21/8354/s1, Figure S1: Species selected for the evolutionary analysis, Figures S2-S24: Phylogenetic reconstruction of genes related to Pi starvation response, Figure S25: Phenotypical response to Pi availability on marchantia polymorpha, Figure S26: Thallus promotes media acidification under low Pi availability, Figure S27: Transcriptional regulation of genes involved in organic acid 4 biosynthesis and exudation, Figure S28: Transcriptional regulation of genes involved in lipid turnover, Figure S29: Transcriptional regulation of peroxidases (MpPRXs) genes, Figure S30: Transcriptional regulation of NADPH oxidase (MpNOXs) genes, Figure S31: Transcriptional regulation of genes related to aronidin biosynthesis and Figure S32: Table for qPCR primers. Table S1: Gene ontology analysis of up-regulated genes at $12 \mathrm{HPT}$, Table S2: Gene ontology analysis of up-regulated genes at $24 \mathrm{HPT}$, Table S3: Gene ontology analysis of up-regulated genes at 150 HPT and Table S4: Enrichment analysis of P1BS motives.

Author Contributions: F.R.-R., M.A.A.-V., L.H.-E. and A.C.-R. conceived the project. F.R.-R., L.H.-E. and A.C.-R. designed the experiments. F.R.-R., S.A.C.-P., A.E.-C., M.D.-Á. and A.O.-A. performed the experiments. F.R.-R., S.A.C.-P., A.E.-C., M.D.-Á., E.H.-B., K.I., M.A.A.-V., and A.C.-R. analyzed the data. K.I., A.C.-H., J.L.B., M.A.A.-V., L.H.-E. and A.C.-R. contributed key bioinformatic resources, biological materials, and reagents. F.R.-R. and A.C.-R. wrote the manuscript with inputs from all co-authors. All authors have read and agreed to the published version of the manuscript.

Funding: F.R.-R. is indebted to Consejo Nacional de Ciencia y Tecnología (CONACyT) for the PhD scholarship (No. 629985). CONACyT provided the PhD scholarship to ACP (No. 420770) and MDA (No. 487660). This work was funded by JSPS KAKENHI grant (19H03247) to K.I. MAAV was funded by UCMEXUS (No. 19941-44), CONACYT Ciencia Básica (No. 158550 \& A1-S-38383) and Royal Society Newton Advanced Fellowship (NA150181).

Acknowledgments: Authors wish to thank T. Kochi from Graduate School of Biostudies at Kyoto University in Japan, for sharing key biological materials. We thank the undergrad student Ambar Jimenez for technical support in standardizing the Pi quantification method. 
Conflicts of Interest: The authors declare that they have no conflict of interest.

\section{Abbreviations}

\begin{tabular}{ll}
$\mathrm{Pi}$ & Phosphate \\
$\mathrm{P} 1 \mathrm{BS}$ & PHR1 binding sites \\
$\mathrm{Ca}$ & Calcium \\
$\mathrm{Al}$ & Aluminium \\
$\mathrm{Fe}$ & Iron \\
$\mathrm{RSA}$ & Root system architecture \\
$\mathrm{OAs}$ & Organic acids \\
$\mathrm{TF}$ & Transcription factor \\
$\mathrm{IPP}$ & Inositol polyphosphate \\
$\mathrm{PM}$ & Plasma membrane \\
+ Pi & High phosphate availability \\
$-\mathrm{Pi}$ & Low phosphate availability \\
$\mathrm{PSR}$ & Phosphate starvation response \\
$\mu \mathrm{M}$ & Micromolar \\
$\mathrm{HAS}$ & Hours after sown \\
$\mathrm{HPT}$ & Hours post transference \\
$\mathrm{DAS}$ & Days after sown \\
FC & Fold change \\
DEGs & Differential expressed genes \\
FDR & False discovery rate \\
GO & Gene ontology \\
qPCR & Quantitative polymerase chain reaction \\
CREs & Cis regulatory elements \\
& \\
\hline
\end{tabular}

\section{References}

1. Sanderson, M.J. Molecular data from 27 proteins do not support a Precambrian origin of land plants. Am. J. Bot. 2003, 90, 954-956. [CrossRef] [PubMed]

2. Delwiche, C.F.; Cooper, E.D. The evolutionary origin of a terrestrial flora. Curr. Biol. 2015, 25, R899-R910. [CrossRef] [PubMed]

3. Pires, N.D.; Dolan, L. Morphological evolution in land plants: New designs with old genes. Philos. Trans. R. Soc. B 2012, 367, 508-518. [CrossRef] [PubMed]

4. Ishizaki, K. Evolution of land plants: Insights from molecular studies on basal lineages. Biosci. Biotechnol. Biochem. 2017, 81, 73-80. [CrossRef]

5. Dipp-Álvarez, M.; Cruz-Ramírez, A. A phylogenetic study of the ANT Family points to a preANT gene as the ancestor of basal and euANT transcription factors in land plants. Front. Plant Sci. 2019, 10, 17. [CrossRef] [PubMed]

6. López-Bucio, J.; Cruz-Ramirez, A.; Herrera-Estrella, L. The role of nutrient availability in regulating root architecture. Curr. Opin. Plant Biol. 2003, 6, 280-287. [CrossRef]

7. Puga, M.I.; Rojas-Triana, M.; de Lorenzo, L.; Leyva, A.; Rubio, V.; Paz-Ares, J. Novel signals in the regulation of Pi starvation responses in plants: Facts and promises. Curr. Opin. Plant Biol. 2017, 39, 40-49. [CrossRef]

8. Gutiérrez-Alanís, D.; Ojeda-Rivera, J.O.; Yong-Villalobos, L.; Cárdenas-Torres, L.; Herrera-Estrella, L. Adaptation to phosphate scarcity: Tips from arabidopsis roots. Trends Plant Sci. 2018, 23, 721-730. [CrossRef]

9. Raghothama, K.G. Phosphate acquisition. Annu. Rev. Plant Biol. 1999, 50, 665-693. [CrossRef]

10. Bieleski, R.L. Phosphate pools, phosphate transport, and phosphate availability. Annu. Rev. Plant Physiol. 1973, 24, 225-252. [CrossRef]

11. Marschner, H. Marschner's Mineral Nutrition of Higher Plants; Academic Press: Amsterdam, The Netherlands, 2011.

12. Lynch, J. Root architecture and plant productivity. Plant Physiol. 1995, 109, 7. [CrossRef] [PubMed] 
13. Thibaud, M.C.; Arrighi, J.F.; Bayle, V.; Chiarenza, S.; Creff, A.; Bustos, R.; Nussaume, L. Dissection of local and systemic transcriptional responses to phosphate starvation in Arabidopsis. Plant J. 2010, 64, 775-789. [CrossRef] [PubMed]

14. Svistoonoff, S.; Creff, A.; Reymond, M.; Sigoillot-Claude, C.; Ricaud, L.; Blanchet, A.; Desnos, T. Root tip contact with low-phosphate media reprograms plant root architecture. Nat. Genet. 2007, 39, 792. [CrossRef] [PubMed]

15. Balzergue, C.; Dartevelle, T.; Godon, C.; Laugier, E.; Meisrimler, C.; Teulon, J.M.; Müller, J. Low phosphate activates STOP1-ALMT1 to rapidly inhibit root cell elongation. Nat. Commun. 2017, 8, 15300. [CrossRef] [PubMed]

16. Mora-Macías, J.; Ojeda-Rivera, J.O.; Gutiérrez-Alanís, D.; Yong-Villalobos, L.; Oropeza-Aburto, A.; Raya-González, J.; Herrera-Estrella, L. Malate-dependent Fe accumulation is a critical checkpoint in the root developmental response to low phosphate. Proc. Natl. Acad. Sci. USA 2017, 114, E3563-E3572. [CrossRef]

17. Zhang, Y.; Zhang, J.; Guo, J.; Zhou, F.; Singh, S.; Xu, X.; Huang, C.F. F-box protein RAE1 regulates the stability of the aluminum-resistance transcription factor STOP1 in Arabidopsis. Proc. Natl. Acad. Sci. USA 2019, 116, 319-327. [CrossRef]

18. Kochian, L.V.; Piñeros, M.A.; Liu, J.; Magalhaes, J.V. Plant adaptation to acid soils: The molecular basis for crop aluminum resistance. Annu. Rev. Plant Biol. 2015, 66, 571-598. [CrossRef]

19. Müller, J.; Toev, T.; Heisters, M.; Teller, J.; Moore, K.L.; Hause, G.; Abel, S. Iron-dependent callose deposition adjusts root meristem maintenance to phosphate availability. Dev. Cell 2015, 33, 216-230. [CrossRef]

20. Song, S.K.; Hofhuis, H.; Lee, M.M.; Clark, S.E. Key divisions in the early Arabidopsis embryo require POL and PLL1 phosphatases to establish the root stem cell organizer and vascular axis. Dev. Cell 2008, 15, 98-109. [CrossRef]

21. Gutiérrez-Alanís, D.; Yong-Villalobos, L.; Jiménez-Sandoval, P.; Alatorre-Cobos, F.; Oropeza-Aburto, A.; Mora-Macías, J.; Herrera-Estrella, L. Phosphate starvation-dependent iron mobilization induces CLE14 expression to trigger root meristem differentiation through CLV2/PEPR2 signaling. Dev. Cell 2017, 41, 555-570. [CrossRef] [PubMed]

22. Rubio, V.; Linhares, F.; Solano, R.; Martín, A.C.; Iglesias, J.; Leyva, A.; Paz-Ares, J. A conserved MYB transcription factor involved in phosphate starvation signaling both in vascular plants and in unicellular algae. Genes Dev. 2001, 15, 2122-2133. [CrossRef]

23. Wild, R.; Gerasimaite, R.; Jung, J.Y.; Truffault, V.; Pavlovic, I.; Schmidt, A.; Mayer, A. Control of eukaryotic phosphate homeostasis by inositol polyphosphate sensor domains. Science 2016, 352, 986-990. [CrossRef]

24. Shimogawara, K.; Wykoff, D.D.; Usuda, H.; Grossman, A.R. Chlamydomonas reinhardtii mutants abnormal in their responses to phosphorus deprivation. Plant Physiol. 1999, 120, 685-694. [CrossRef]

25. Wykoff, D.D.; Grossman, A.R.; Weeks, D.P.; Usuda, H.; Shimogawara, K. Psr1, a nuclear localized protein that regulates phosphorus metabolism in Chlamydomonas. Proc. Natl. Acad. Sci. USA 1999, 96, 15336-15341. [CrossRef]

26. Nussaume, L.; Kanno, S.; Javot, H.; Marin, E.; Nakanishi, T.M.; Thibaud, M.C. Phosphate import in plants: Focus on the PHT1 transporters. Front. Plant Sci. 2011, 2, 83. [CrossRef]

27. Kanno, S.; Arrighi, J.F.; Chiarenza, S.; Bayle, V.; Berthomé, R.; Péret, B.; Thibaud, M.C. A novel role for the root cap in phosphate uptake and homeostasis. Elife 2016, 5, e14577. [CrossRef]

28. Daram, P.; Brunner, S.; Rausch, C.; Steiner, C.; Amrhein, N.; Bucher, M. Pht2; 1 encodes a low-affinity phosphate transporter from Arabidopsis. Plant Cell 1999, 11, 2153-2166. [CrossRef] [PubMed]

29. Versaw, W.K.; Harrison, M.J. A chloroplast phosphate transporter, PHT2; 1, influences allocation of phosphate within the plant and phosphate-starvation responses. Plant Cell 2002, 14, 1751-1766. [CrossRef]

30. Guo, B.; Irigoyen, S.; Fowler, T.B.; Versaw, W.K. Differential expression and phylogenetic analysis suggest specialization of plastid-localized members of the PHT4 phosphate transporter family for photosynthetic and heterotrophic tissues. Plant Signal. Behav. 2008, 3, 784-790. [CrossRef] [PubMed]

31. Guo, B.; Jin, Y.; Wussler, C.; Blancaflor, E.B.; Motes, C.M.; Versaw, W.K. Functional analysis of the Arabidopsis PHT4 family of intracellular phosphate transporters. New Phytol. 2008, 177, 889-898. [CrossRef] [PubMed]

32. Takabatake, R.; Hata, S.; Taniguchi, M.; Kouchi, H.; Sugiyama, T.; Izui, K. Isolation and characterization of cDNAs encoding mitochondrial phosphate transporters in soybean, maize, rice, and Arabidopsis. Plant Mol. Biol. 1999, 40, 479-486. [CrossRef] [PubMed] 
33. Jia, F.; Wan, X.; Zhu, W.; Sun, D.; Zheng, C.; Liu, P.; Huang, J. Overexpression of mitochondrial phosphate transporter 3 severely hampers plant development through regulating mitochondrial function in Arabidopsis. PLoS ONE 2015, 10, e0129717. [CrossRef]

34. Liu, J.; Yang, L.; Luan, M.; Wang, Y.; Zhang, C.; Zhang, B.; Luan, S. A vacuolar phosphate transporter essential for phosphate homeostasis in Arabidopsis. Proc. Natl. Acad. Sci. USA 2015, 112, E6571-E6578. [CrossRef] [PubMed]

35. Xu, L.; Zhao, H.; Wan, R.; Liu, Y.; Xu, Z.; Tian, W.; Dolan, L. Identification of vacuolar phosphate efflux transporters in land plants. Nat. Plants 2019, 5, 84. [CrossRef]

36. Essigmann, B.; Güler, S.; Narang, R.A.; Linke, D.; Benning, C. Phosphate availability affects the thylakoid lipid composition and the expression of SQD1, a gene required for sulfolipid biosynthesis in Arabidopsis thaliana. Proc. Natl. Acad. Sci. USA 1998, 95, 1950-1955. [CrossRef]

37. Cruz-Ramírez, A.; Oropeza-Aburto, A.; Razo-Hernández, F.; Ramírez-Chávez, E.; Herrera-Estrella, L. Phospholipase DZ2 plays an important role in extraplastidic galactolipid biosynthesis and phosphate recycling in Arabidopsis roots. Proc. Natl. Acad. Sci. USA 2006, 103, 6765-6770.

38. Andersson, M.X.; Stridh, M.H.; Larsson, K.E.; Liljenberg, C.; Sandelius, A.S. Phosphate-deficient oat replaces a major portion of the plasma membrane phospholipids with the galactolipid digalactosyldiacylglycerol. FEBS Lett. 2003, 537, 128-132. [CrossRef]

39. Bariola, P.A.; Howard, C.J.; Taylor, C.B.; Verburg, M.T.; Jaglan, V.D.; Green, P.J. The Arabidopsis ribonuclease gene RNS1 is tightly controlled in response to phosphate limitation. Plant J. 1994, 6, 673-685. [CrossRef]

40. Li, D.; Zhu, H.; Liu, K.; Liu, X.; Leggewie, G.; Udvardi, M.; Wang, D. Purple acid phosphatases of Arabidopsis thaliana comparative analysis and differential regulation by phosphate deprivation. J. Biol. Chem. 2002, 277, 27772-27781. [CrossRef]

41. Puga, M.I.; Mateos, I.; Charukesi, R.; Wang, Z.; Franco-Zorrilla, J.M.; de Lorenzo, L.; Leyva, A. SPX1 is a phosphate-dependent inhibitor of Phosphate Starvation Response 1 in Arabidopsis. Proc. Natl. Acad. Sci. USA 2014, 111, 14947-14952. [CrossRef] [PubMed]

42. Wang, Z.; Ruan, W.; Shi, J.; Zhang, L.; Xiang, D.; Yang, C.; Shou, H. Rice SPX1 and SPX2 inhibit phosphate starvation responses through interacting with PHR2 in a phosphate-dependent manner. Proc. Natl. Acad. Sci. USA 2014, 111, 14953-14958. [CrossRef] [PubMed]

43. Lv, Q.; Zhong, Y.; Wang, Y.; Wang, Z.; Zhang, L.; Shi, J.; Wu, P. SPX4 negatively regulates phosphate signaling and homeostasis through its interaction with PHR2 in rice. Plant Cell 2014, 26, 1586-1597. [CrossRef]

44. Osorio, M.B.; Ng, S.; Berkowitz, O.; De Clercq, I.; Mao, C.; Shou, H.; Jost, R. SPX4 Acts on PHR1-Dependent and-Independent Regulation of Shoot Phosphorus Status in Arabidopsis. Plant Physiol. 2019, 332-352. [CrossRef] [PubMed]

45. Shull, C. Phosphorus and the early maturity of plants. Trans. Illinois State Acad. Sci. 1925, 18, $165-171$.

46. Voth, P.D.; Hamner, K.C. Responses of Marchantia polymorpha to nutrient supply and photoperiod. Bot. Gaz. 1940, 102, 169-205. [CrossRef]

47. Voth, P.D. Gemmae-cup production in Marchantia polymorpha and its response to calcium deficiency and supply of other nutrients. Bot. Gaz. 1941, 103, 310-325. [CrossRef]

48. Bonnot, C.; Proust, H.; Pinson, B.; Colbalchini, F.P.; Lesly-Veillard, A.; Breuninger, H.; Dolan, L. Functional PTB phosphate transporters are present in streptophyte algae and early diverging land plants. New Phytol. 2017, 214, 1158-1171. [CrossRef] [PubMed]

49. Shimamura, M. Marchantia polymorpha: Taxonomy, phylogeny and morphology of a model system. Plant Cell Physiol. 2016, 57, 230-256. [CrossRef] [PubMed]

50. Bowman, J.L.; Kohchi, T.; Yamato, K.T.; Jenkins, J.; Shu, S.; Ishizaki, K.; Adam, C. Insights into land plant evolution garnered from the Marchantia polymorpha genome. Cell 2017, 171, 287-304. [CrossRef]

51. Péret, B.; Clément, M.; Nussaume, L.; Desnos, T. Root developmental adaptation to phosphate starvation: Better safe than sorry. Trends Plant Sci. 2011, 16, 442-450. [CrossRef]

52. Albert, N.W.; Thrimawithana, A.H.; McGhie, T.K.; Clayton, W.A.; Deroles, S.C.; Schwinn, K.E.; Davies, K.M. Genetic analysis of the liverwort Marchantia polymorpha reveals that R2R3 MYB activation of flavonoid production in response to abiotic stress is an ancient character in land plants. New Phytol. 2018, 218, 554-566. [CrossRef] 
53. Berland, H.; Albert, N.W.; Stavland, A.; Jordheim, M.; McGhie, T.K.; Zhou, Y.; Davies, K.M. Auronidins are a previously unreported class of flavonoid pigments that challenges when anthocyanin biosynthesis evolved in plants. Proc. Natl. Acad. Sci. USA 2019, 116, 20232-20239. [CrossRef]

54. Del Pozo, J.C.; Allona, I.; Rubio, V.; Leyva, A.; De La Peña, A.; Aragoncillo, C.; Paz-Ares, J. A type 5 acid phosphatase gene from Arabidopsis thaliana is induced by phosphate starvation and by some other types of phosphate mobilising/oxidative stress conditions. Plant J. 1999, 19, 579-589. [CrossRef]

55. Huang, Z.; Terpetschnig, E.; You, W.; Haugland, R.P. 2-(2'-Phosphoryloxyphenyl)-4 (3H)-quinazolinone derivatives as fluorogenic precipitating substrates of phosphatases. Anal. Biochem. 1992, 207, 32-39. [CrossRef]

56. Misson, J.; Raghothama, K.G.; Jain, A.; Jouhet, J.; Block, M.A.; Bligny, R.; Doumas, P. A genome-wide transcriptional analysis using Arabidopsis thaliana Affymetrix gene chips determined plant responses to phosphate deprivation. Proc. Natl. Acad. Sci. USA 2005, 102, 11934-11939. [CrossRef]

57. Secco, D.; Jabnoune, M.; Walker, H.; Shou, H.; Wu, P.; Poirier, Y.; Whelan, J. Spatio-temporal transcript profiling of rice roots and shoots in response to phosphate starvation and recovery. Plant Cell 2013, 25, 4285-4304. [CrossRef]

58. Calderón-Vázquez, C.; Sawers, R.J.; Herrera-Estrella, L. Phosphate deprivation in maize: Genetics and genomics. Plant Physiol. 2011, 156, 1067-1077. [CrossRef]

59. Zeng, H.; Wang, G.; Zhang, Y.; Hu, X.; Pi, E.; Zhu, Y.; Du, L. Genome-wide identification of phosphate-deficiency-responsive genes in soybean roots by high-throughput sequencing. Plant Soil 2016, 398, 207-227. [CrossRef]

60. Bindea, G.; Mlecnik, B.; Hackl, H.; Charoentong, P.; Tosolini, M.; Kirilovsky, A.; Galon, J. ClueGO: A Cytoscape plug-in to decipher functionally grouped gene ontology and pathway annotation networks. Bioinformatics 2009, 25, 1091-1093. [CrossRef]

61. Hirakawa, Y.; Uchida, N.; Yamaguchi, Y.L.; Tabata, R.; Ishida, S.; Ishizaki, K.; Bowman, J.L. Control of proliferation in the haploid meristem by CLE peptide signaling in Marchantia polymorpha. PLoS Genet. 2019, 15, e1007997. [CrossRef]

62. Hirakawa, Y.; Fujimoto, T.; Ishida, S.; Uchida, N.; Sawa, S.; Kiyosue, T.; Bowman, J.L. Induction of Multichotomous Branching by CLAVATA Peptide in Marchantia polymorpha. Curr. Biol. 2020. [CrossRef] [PubMed]

63. Liu, J.; Magalhaes, J.V.; Shaff, J.; Kochian, L.V. Aluminum-activated citrate and malate transporters from the MATE and ALMT families function independently to confer Arabidopsis aluminum tolerance. Plant J. 2019, 57, 389-399. [CrossRef]

64. Mittler, R.; Vanderauwera, S.; Suzuki, N.; Miller, G.A.D.; Tognetti, V.B.; Vandepoele, K.; Van Breusegem, F. ROS signaling: The new wave? Trends Plant Sci. 2011, 16, 300-309. [CrossRef]

65. Foreman, J.; Demidchik, V.; Bothwell, J.H.; Mylona, P.; Miedema, H.; Torres, M.A.; Davies, J.M. Reactive oxygen species produced by NADPH oxidase regulate plant cell growth. Nature 2003, 422, 442-446. [CrossRef]

66. Carol, R.J.; Takeda, S.; Linstead, P.; Durrant, M.C.; Kakesova, H.; Derbyshire, P.; Dolan, L. A RhoGDP dissociation inhibitor spatially regulates growth in root hair cells. Nature 2005, 438, 1013-1016. [CrossRef]

67. Saint-Marcoux, D.; Proust, H.; Dolan, L.; Langdale, J.A. Identification of reference genes for real-time quantitative PCR experiments in the liverwort Marchantia polymorpha. PLoS ONE 2015, 10, e0118678. [CrossRef]

68. Bustos, R.; Castrillo, G.; Linhares, F.; Puga, M.I.; Rubio, V.; Pérez-Pérez, J.; Paz-Ares, J. A central regulatory system largely controls transcriptional activation and repression responses to phosphate starvation in Arabidopsis. PLoS Genet. 2010, 6, e1001102. [CrossRef]

69. McLeay, R.C.; Bailey, T.L. Motif Enrichment Analysis: A unified framework and an evaluation on ChIP data. BMC Bioinform. 2010, 11, 165. [CrossRef]

70. Bailey, T.L.; Boden, M.; Buske, F.A.; Frith, M.; Grant, C.E.; Clementi, L.; Noble, W.S. MEME SUITE: Tools for motif discovery and searching. Nucleic Acids Res. 2009, 37 (Suppl. 2), W202-W208. [CrossRef] [PubMed]

71. Castrillo, G.; Teixeira, P.J.P.L.; Paredes, S.H.; Law, T.F.; de Lorenzo, L.; Feltcher, M.E.; Paz-Ares, J. Root microbiota drive direct integration of phosphate stress and immunity. Nature 2017, 543, 513-518. [CrossRef] 
72. Briat, J.F.; Rouached, H.; Tissot, N.; Gaymard, F.; Dubos, C. Integration of P, S, Fe, and Zn nutrition signals in Arabidopsis thaliana: Potential involvement of PHOSPHATE STARVATION RESPONSE 1 (PHR1). Front. Plant Sci. 2015, 6, 290. [CrossRef]

73. Li, W.; Lan, P. Genome-wide analysis of overlapping genes regulated by iron deficiency and phosphate starvation reveals new interactions in Arabidopsis roots. BMC Res. Notes 2015, 8, 555. [CrossRef]

74. Kato, H.; Ishizaki, K.; Kouno, M.; Shirakawa, M.; Bowman, J.L.; Nishihama, R.; Kohchi, T. Auxin-mediated transcriptional system with a minimal set of components is critical for morphogenesis through the life cycle in Marchantia polymorpha. PLoS Genet. 2015, 11, e1005084. [CrossRef]

75. Flores-Sandoval, E.; Eklund, D.M.; Bowman, J.L. A simple auxin transcriptional response system regulates multiple morphogenetic processes in the liverwort Marchantia polymorpha. PLoS Genet. 2015, 11, e1005207. [CrossRef] [PubMed]

76. López-Bucio, J.; Hernández-Abreu, E.; Sánchez-Calderón, L.; Nieto-Jacobo, M.F.; Simpson, J.; Herrera-Estrella, L. Phosphate availability alters architecture and causes changes in hormone sensitivity in the Arabidopsis root system. Plant Physiol. 2002, 129, 244-256. [CrossRef]

77. Huang, K.L.; Ma, G.J.; Zhang, M.L.; Xiong, H.; Wu, H.; Zhao, C.Z.; Li, X.B. The ARF7 and ARF19 transcription factors positively regulate PHOSPHATE STARVATION RESPONSE1 in Arabidopsis roots. Plant Physiol. 2018, 178, 413-427. [CrossRef]

78. Clayton, W.A.; Albert, N.W.; Thrimawithana, A.H.; McGhie, T.K.; Deroles, S.C.; Schwinn, K.E.; Davies, K.M. UVR8-mediated induction of flavonoid biosynthesis for UVB tolerance is conserved between the liverwort Marchantia polymorpha and flowering plants. Plant J. 2018, 96, 503-517. [CrossRef]

79. Kubo, H.; Nozawa, S.; Hiwatashi, T.; Kondou, Y.; Nakabayashi, R.; Mori, T.; Ishizaki, K. Biosynthesis of riccionidins and marchantins is regulated by R2R3-MYB transcription factors in Marchantia polymorpha. J. Plant Res. 2018, 131, 849-864. [CrossRef]

80. Moseley, J.; Grossman, A.R. Phosphate metabolism and responses to phosphorus deficiency. In The Chlamydomonas Sourcebook; Harris, E.H., Ed.; Academic Press: Amsterdam, The Netherlands, 2009; pp. 189-215.

81. González-Muñoz, E.; Avendaño-Vázquez, A.O.; Montes, R.A.C.; de Folter, S.; Andrés-Hernández, L.; Abreu-Goodger, C.; Sawers, R.J. The maize (Zea mays ssp. mays var. B73) genome encodes 33 members of the purple acid phosphatase family. Front. Plant Sci. 2015, 6, 341.

82. Mehra, P.; Pandey, B.K.; Giri, J. Improvement in phosphate acquisition and utilization by a secretory purple acid phosphatase (OsPAP21b) in rice. Plant Biotechnol. J. 2017, 15, 1054-1067. [CrossRef]

83. Reinhard, C.T.; Planavsky, N.J.; Gill, B.C.; Ozaki, K.; Robbins, L.J.; Lyons, T.W.; Konhauser, K.O. Evolution of the global phosphorus cycle. Nature 2017, 541, 386-389. [CrossRef] [PubMed]

84. Alcaraz, L.D.; Olmedo, G.; Bonilla, G.; Cerritos, R.; Hernández, G.; Cruz, A.; López, V. The genome of Bacillus coahuilensis reveals adaptations essential for survival in the relic of an ancient marine environment. Proc. Natl. Acad. Sci. USA 2008, 105, 5803-5808. [CrossRef]

85. Gargouri, M.; Park, J.J.; Holguin, F.O.; Kim, M.J.; Wang, H.; Deshpande, R.R.; Gang, D.R. Identification of regulatory network hubs that control lipid metabolism in Chlamydomonas reinhardtii. J. Exp. Bot. 2015, 66, 4551-4566. [CrossRef]

86. Kato, H.; Mutte, S.K.; Suzuki, H.; Crespo, I.; Das, S.; Radoeva, T.; Lindhoud, S. Design principles of a minimal auxin response system. Nat. Plants 2020, 6, 473-482. [CrossRef]

87. Höwing, T.; Huesmann, C.; Hoefle, C.; Nagel, M.K.; Isono, E.; Huckelhoven, R.; Gietl, C. Endoplasmic reticulum KDEL-tailed cysteine endopeptidase 1 of Arabidopsis (AtCEP1) is involved in pathogen defense. Front. Plant Sci. 2014, 5, 58. [CrossRef]

88. Altschul, S.F.; Madden, T.L.; Schäffer, A.A.; Zhang, J.; Zhang, Z.; Miller, W.; Lipman, D.J. Gapped BLAST and PSI-BLAST: A new generation of protein database search programs. Nucleic Acids Res. 1997, 25, 3389-3402. [CrossRef]

89. Katoh, K.; Standley, D.M. MAFFT multiple sequence alignment software version 7: Improvements in performance and usability. Mol. Biol. Evol. 2013, 30, 772-780. [CrossRef]

90. Capella-Gutiérrez, S.; Silla-Martínez, J.M.; Gabaldón, T. trimAl: A tool for automated alignment trimming in large-scale phylogenetic analyses. Bioinformatics 2009, 25, 1972-1973. [CrossRef]

91. Posada, D.; Crandall, K.A. Modeltest: Testing the model of DNA substitution. Bioinformatics 1998, 14, 817-818. [CrossRef] 
92. Miller, M.A.; Pfeiffer, W.; Schwartz, T. Creating the CIPRES Science Gateway for inference of large phylogenetic trees. In Proceedings of the 2010 Gateway Computing Environments Workshop (GCE), New Orleans, LA, USA, 14 November 2010; pp. 1-8.

93. Letunic, I.; Bork, P. Interactive Tree Of Life (iTOL) v4: Recent updates and new developments. Nucleic Acids Res. 2019, 47, W256-W259. [CrossRef] [PubMed]

94. Bolger, A.M.; Lohse, M.; Usadel, B. Trimmomatic: A flexible trimmer for Illumina sequence data. Bioinformatics 2014, 30, 2114-2120. [CrossRef]

95. Heinz, S.; Benner, C.; Spann, N.; Bertolino, E.; Lin, Y.C.; Laslo, P.; Cheng, J.X.; Murre, C.; Singh, H.; Glass, C.K. Simple Combinations of Lineage-Determining Transcription Factors Prime cis-Regulatory Elements Required for Macrophage and B Cell Identities. Mol. Cell 2010. [CrossRef]

96. Wagih, O. ggseqlogo: A versatile R package for drawing sequence logos. Bioinformatics 2017. [CrossRef] [PubMed]

Publisher's Note: MDPI stays neutral with regard to jurisdictional claims in published maps and institutional affiliations.

(C) 2020 by the authors. Licensee MDPI, Basel, Switzerland. This article is an open access article distributed under the terms and conditions of the Creative Commons Attribution (CC BY) license (http://creativecommons.org/licenses/by/4.0/). 